\title{
Utilisation combinée des deux sources de rayonnements laser et synchrotron : caractéristiques et résultats
}

\author{
F.J. Wuilleumier, D. Cubaynes et J.-M. Bizau \\ Laboratoire de Spectroscopie Atomique et Ionique, Université Paris-Sud, Bàtiment 350, \\ 91405 Orsay Cedex, France
}

\section{ABSTRACT.}

The first experiments combining the use of $\mathrm{cw}$ dye lasers with synchrotron radiation for inner-shell photoionization in laser-excited atoms, were carried out more than 10 years ago. Since that time, a number of resonant inner-shell photoexcitation processes have been measured. The results of these measurements will be summarized. The use of new synchrotron radiation sources such as the third generation storage ring Super ACO in Orsay or the undulator installed at BESSY, in Berlin, enhanced the photon flux available, making feasible new experiments. At high atomic densities, partial photoionization cross sections or angular distribution of inner-electrons were measured in laser-excited alkali- and alkaline earth atoms, revealing strong enhancements of the intensity of correlation satellites in inner-shell photoionization of excited atoms. At low atomic densities, synchrotron radiation studies of laser-aligned atoms were started, allowing to demonstrate the influence of the laser polarization on the angular distribution of electrons emitted in the decay of core-excited autoionizing states. These new results will be described. Finally, the perspectives open for present and future uses of third generation storage rings in combination with laser(s) will be discussed.

\section{INTRODUCTION}

L'idée de combiner l'utilisation d'un rayonnement laser avec celle du rayonnement synchrotron a été initiée à Orsay et les premières expériences démontrant la faisabilité d'études de photoionisation sur des atomes excités avec ces deux sources ont été couronnées de succès dès 1981 dans le cas du sodium excité[1] dans l'état $2 \mathrm{p}^{6} 3 \mathrm{p}^{2} \mathrm{P}_{3 / 2}$ et 1982 pour le baryum excité[2] dans les états $5 \mathrm{p}^{6} 6 \mathrm{~s} 5 \mathrm{~d}^{1,3} \mathrm{D}_{2}$.

La première utilisation synchronisée de deux sources de photons pour étudier un système atomique dans un état différent de l'état fondamental de l'atome neutre (atomes excités ou ionisés), remonte[3,4], à notre connaissance, à 1969. La synchronisation temporelle de deux étincelles émises par décharge sous vide, l'une servant à produire le milieu absorbant, l'autre fournissant le rayonnement nécessaire à l'étude de la photoabsorption de ce milieu, conduisit aux premières études sur des espèces ioniques, d'abord monochargées[3], puis doublement chargées[5,6], d'atomes alcalino-terreux. Mais les progrès décisifs vinrent de la mise en oeuvre de lasers pulsés. La séparation en deux faisceaux du rayonnement émis par un laser à verre néodyme et leurs focalisation synchronisée sur deux cibles solides différentes permirent à Jaeglé et son équipe[7] de démarrer un programme de recherche sur les processus d'émission et d'absorption dans les ions multichargés et d'aboutir, après la mise en évidence d'inversions de populations[8], à la réalisation du premier effet laser dans le domaine des rayons X mous[9]. Cette méthode a parallèlement été développée[10,11] et est maintenant largement utilisée, sous 
le nom de DLPP (Dual Laser Produced Plasmas) pour l'étude systématique des spectres de photoabsorption de nombreux ions multichargés[12-15]. Une variante de cette méthode, intermédiaire entre la technique Mehlman-Esteva et la technique Jaeglé, a associé l'utilisation de la source à décharges BRV, émettant un rayonnement continu entre $30 \mathrm{eV}$ et $100 \mathrm{eV}$, avec celle d'un laser à colorant pulsé accordé à l'énergie de la première transition d'excitation résonante d'un électron externe dans un atome ou un ion monochargé d'alcalins ou d'alcalinoterreux[16-18]. Les collisions entre atomes ou ions excités produisent rapidement, pourvu que la densité soit suffisamment élevée $\left(\approx 10^{15}\right.$ atomes $\left./ \mathrm{cm}^{3}\right)$ l'excitation ou l'ionisation complète du milieu, suivant le retard temporel introduit dans l'émission des deux sources de photons. Cette méthode, connue sous le nom RLDI (Resonant Laser Driven Ionization), fut à l'origine des premières études portant sur la photoabsorption, en couche interne, d'atomes alcalins excités en couche externe[17, 19] et d'ions monochargés alcalins et alcalino-terreux[16-18]. Elle a été raffinée plus récemment avec le remplacement de la source BRV par un laser pulsé, à verre néodyme ou à rubis, pour produire, par focalisation sur une cible solide, le rayonnement continu VUV servant à "sonder" le milieu excité ou ionisé[20-22].

En ce qui concerne plus spécifiquement les états excités, d'autres modes ont été testés pour la partie "pompe" de ces expériences, dites "pompe-sonde", la sonde étant, dans la plupart des cas, un laser pulsé. Différentes techniques ont été successivement essayées: un laser résonant[23,24], des lampes[25,26], un faisceau d'électrons[27,28]. La première mesure d'une section efficace de photoionisation en couche externe d'un atome excité a combiné un faisceau d'électrons pour produire un état excité de l'hélium (avec une faible population) et un faisceau laser pour ioniser l'électron ainsi excité[27]. Plus, tard, les progrès accomplis dans le développement des lasers ont permis des études[29-33] de distribution angulaire, de mesure du spin et de l'alignement des états magnétiques, toujours dans le cas de l'ionisation de l'électron excité. Mais si la "sonde" est un laser, et elle l'a été pour la plupart de ces expériences (à une exception près[33]), seule la photoionisation en couche externe de ces atomes excités peut être testée et seulement dans un domaine d'énergie très étroit, au plus de quelques électron-volts au dessus de la première limite d'ionisation, étant donné le faible domaine spectral couvert par les lasers actuellement disponibles. L'utilisation du rayonnement synchrotron comme source de photons ionisants a permis d'aborder l'étude de ces atomes par spectrométrie d'électrons et d'étendre sur plus de $100 \mathrm{eV}$ le domaine d'énergie de photons, ouvrant particulièrement la voie à l'exploration des processus de photoexcitation (résonants)[34,35], puis de photoionisation (dans le continuum)[36-37] d'électrons appartenant à une couche interne.

Plus récemment, l'influence de l'alignement des atomes excités induit par la polarisation du faisceau laser à des densités atomiques plus faibles a été testée[38], puis plus systématiquement étudiée[39] pour le lithium grâce à l'utilisation du rayonnement synchrotron émis par un onduleur. Enfin, l'application de ces techniques en physique moléculaire a commencé récemment par l'étude des fragments, atomiques ou moléculaires, produits dans leur état fondamental par la dissociation de molécules à l'aide d'un laser continu[40]. La combinaison des techniques laser et synchrotron pour la photoionisation de molécules préparées dans un état excité n'a pas encore été réalisée, à la différence des techniques associant deux ou plusieurs lasers pulsés, bien qu'elle soit au centre de nombreux projets prévoyant d'utiliser des anneaux de stockage de la troisième génération associés, en particulier, à un laser à électrons libres fonctionnant dans l'infrarouge[41]. L'application de ces techniques en physique moléculaire est revue dans un autre chapitre de ce volume par I. Nenner et nous n'en parlerons donc pas dans ce texte.

Dans la suite de cette brève revue, nous présenterons les techniques mises au point et résumerons les résultats qui ont pu être obtenus en physique atomique et qui concernent la photoionisation résonante (autoionisation) et la photoionisation directe dans le continuum, en couche interne, d'atomes alcalins et alcalino-terreux excités en couche externe. 


\section{CARACTERISTIQUES DES RAYONNEMENTS ET METHODE EXPERIMENTALE}

Nous avons résumé, dans la Table I, les caractéristiques comparées des lasers continus, des lasers pulsés et du rayonnement synchrotron, qui ont conduit aux choix technologiques discutés antérieurement par Wuilleumier et al.[2, 42-45]. Les types de lasers sélectionnés pour cette comparaison sont des lasers disponibles commercialement, relativement faciles à mettre en oeuvre dans l'environnement peu favorable d'un centre de rayonnement synchrotron, et les performances rappelées ici ne sont donc pas celles des lasers de pointe optimisés dans les laboratoires de recherche sur les lasers. On remarque, en particulier, que le faible nombre de photons disponibles dans un pulse de rayonnement synchrotron, d'une part, et le faible taux de répétition des lasers pulsés d'autre part, rendent totalement inefficace, pour l'instant, tout essai de combinaison de ces deux types de source en physique atomique. Il en est autrement, par

Table I. Caractéristiques comparées du rayonnement émis par les lasers à colorant et du rayonnement synchrotron

\begin{tabular}{|c|c|c|c|}
\hline Caractéristiques & $\begin{array}{l}\frac{\text { Laser à colorant }}{\text { pulsé }} \\
\text { put }\end{array}$ & $\frac{\text { Laser à colorant }}{\underline{\text { continu }}}$ & $\begin{array}{l}\text { Rayonnement } \\
\text { synchrotron }\end{array}$ \\
\hline $\begin{array}{c}\text { Domaine } \\
\text { d'accordabilité }\end{array}$ & Etroit: 0,5 à $15 \mathrm{eV}$ & Très étroit: 1 à $5 \mathrm{eV}$ & $\begin{array}{c}\text { Très étendu: } \\
\text { quelques eV à } \\
\text { plusieurs dizaines de } \\
\text { keV }\end{array}$ \\
\hline $\begin{array}{c}\text { Résolution } \\
\text { spectrale } \Delta \mathbf{E} / \mathbf{E}\end{array}$ & $\begin{array}{l}\text { Modérée: environ } \\
\qquad 10^{-4}\end{array}$ & $\begin{array}{c}\text { Excellente: aisément } \\
10^{-8} \text { à } 10^{-9}\end{array}$ & $\begin{array}{l}\text { Modérée, dépend du } \\
\text { flux nécessaire: } 10^{-2} \\
\text { à } 10^{-4}\end{array}$ \\
\hline $\begin{array}{l}\text { Intensité par } \\
\text { impulsion ou par } \\
\text { seconde }\end{array}$ & $\begin{array}{l}\text { Peut être très élevée: } \\
10^{16} \text { à } 10^{18} \text { photons/ } \\
\text { impulsion mais avec } \\
\text { un faible taux de } \\
\text { répétition }\end{array}$ & $\begin{array}{l}\text { Elevée: } 10^{18} \text { à } 10^{19} \\
\text { phot‘ns par secc nde }\end{array}$ & $\begin{array}{c}\text { Faible: } 10^{5} \text { à } 10^{6} \\
\text { photons/impulsion d' } \\
\text { environ 1 nsec } \\
10^{12} \text { (sur aimant de } \\
\text { courbure) à } 10^{13} \\
\text { photons/sec (ondu- } \\
\text { leur) dans une bande } \\
\text { passante de } 10^{-3}\end{array}$ \\
\hline $\begin{array}{l}\text { Structure } \\
\text { temporelle }\end{array}$ & $\begin{array}{c}\text { Variable: impulsions } \\
\text { d'une durée de } 10^{-12} \\
\text { à } 10^{-8} \mathrm{sec} \text { avec un } \\
\text { taux de répétition de } \\
1 \text { à } 10^{3} / \mathrm{sec}\end{array}$ & Continue & $\begin{array}{l}\text { Pulsée: impulsions } \\
\text { de } 10^{-10} \text { à } 10^{-9} \mathrm{sec} \\
\text { avec un taux de } \\
\text { répétition de } 10^{7} / \mathrm{sec} \\
\text { (qq. } 10^{4} \text { à qq.10 } \\
\text { photons/impulsion) à } \\
10^{8} / \mathrm{sec}\left(10^{4} \text { à } 10^{5}\right. \\
\text { photons/impulsion) }\end{array}$ \\
\hline Polarisation & $\begin{array}{l}\text { Linéaire ou } \\
\text { circulaire, facile à } \\
\text { changer }\end{array}$ & $\begin{array}{c}\text { Linéaire ou } \\
\text { circulaire, facile à } \\
\text { changer }\end{array}$ & $\begin{array}{l}\text { Difficile à changer } \\
\text { Linéaire dans le plan } \\
\text { de l'orbite, elliptique } \\
\text { en dehors de ce plan, } \\
\text { circulaire avec des } \\
\text { onduleurs spéciaux }\end{array}$ \\
\hline
\end{tabular}


exemple pour des études en photodissociation moléculaire ou en physique du solide, avec l'utilisation de lasers dont la fréquence est asservie à la fréquence de répétition du rayonnement synchrotron[46].

Quant à la combinaison du rayonnement synchrotron avec le rayonnement d'un laser à colorant continu, bien que les caractéristiques de ces deux sources soient, pour la plupart, à l'opposé les unes des autres, elle était et reste la seule possible pour l'étude des états atomiques excités. L'avantage le plus important amené par le rayonnement synchrotron est, bien évidemment, le large domaine d'énergie à l'intérieur duquel il est continûment accordable, alors que celui d'un laser continu réside dans l'intensité élevée disponible dans une largeur spectrale extrêmement fine. La possibilité de jouer avec les polarisations respectives des deux rayonnements a été plus récemment explorée [39] et sera très certainement d'avantage mise en oeuvre dans le futur. En particulier, la réalisation d'onduleurs croisés va permettre de produire du rayonnement synchrotron circulairement polarisé sans avoir, comme jusqu'à maintenant, à se mettre hors du plan de l'orbite, à des angles d'élévation auxquels l'intensité du rayonnement synchrotron est très réduite.

Alors que les techniques de lasers pulsés précédemment utilisées avaient permis d'étudier la photoabsorption d'atomes excités, la grande nouveauté apportée par la combinaison du rayonnement synchrotron avec celui d'un laser à colorant continu, a été de rendre possible des expériences en spectroscopie d'électrons, technique expérimentale qui, seule, permet de mesurer les paramètres dynamiques de la photoionisation: sections efficaces partielles, distribution angulaire, polarisation de spin des photoélectrons. La figure 1 montre le dispositif expérimental utilisé maintenant à Orsay, sur la ligne SA23 de Super ACO, qui, dans son principe reste le même que le dispositif original implanté $[42,43]$ sur ACO en 1981 .

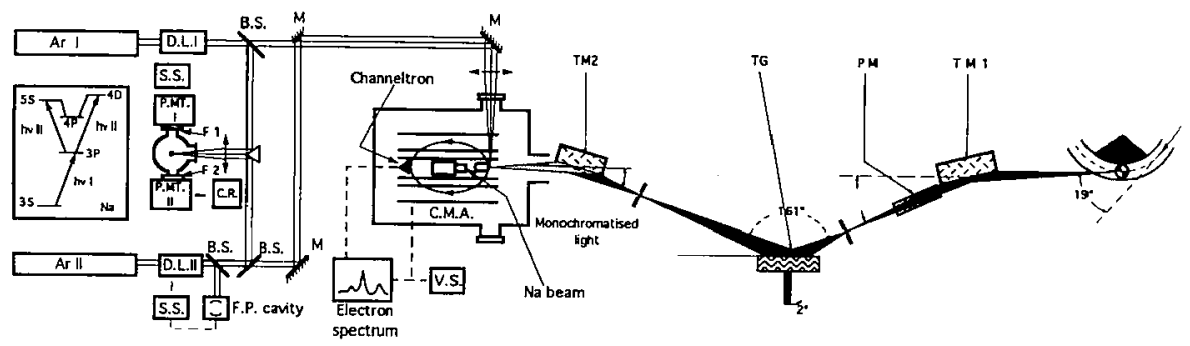
Fig. 1 - Schéma du dispositif expérimental disponible à Super ACO pour l'utilisation
simultanée de rayonnements synchrotron et laser.

Le rayonnement synchrotron émis par le faisceau de positrons dans l'anneau de stockage est d'abord focalisé, dans le plan vertical, par un miroir torique TM1, sur la fente d'entrée d'un monochromateur à réseau torique[47], le rayonnement ayant été également dévié dans le plan horizontal par réflexion sur un miroir plan PM. Le rayonnement synchrotron est ensuite rendu monochromatique par diffraction sur un réseau torique TG (deux réseaux sont disponibles dans l'enceinte et sont interchangeables sous vide, afin de couvrir, dans les conditions les plus optimisées, un large domaine d'énergie de photons, ici de $15 \mathrm{eV}$ à environ $150 \mathrm{eV}$ ), qui a aussi pour effet de le refocaliser sur la fente de sortie du monochromateur. La largeur des fentes d'entrée et de sortie est réglable sous vide de façon continue, caractéristique très importante permettant d'adapter à tout instant la résolution utilisable dans une expérience au flux de photons disponible. Les bandes passantes pratiquement utilisées dans ces expériences ont varié de $0,08 \mathrm{eV}$ à $0,3 \mathrm{eV}$ entre $20 \mathrm{eV}$ et $70 \mathrm{eV}$ et de $0,3 \mathrm{eV}$ à $1,0 \mathrm{eV}$ jusqu'à $120 \mathrm{eV}$. 
Après la fente de sortie, le rayonnement tombe sur un deuxième miroir torique TM2 qui le refocalise dans le volume source d'un analyseur d'électrons, ici un spectromètre miroir cylindrique (CMA). Le CMA offre le meilleur compromis résolution-transmission et ce choix fut déterminant, à l'époque où les flux de photons disponibles étaient plus faibles que maintenant[48]. Les photoélectrons émis dans le volume source de l'analyseur, sont détectés avec un channeltron placé sur l'axe du CMA, après traversée d'un système de quatre fentes (deux à l'entrée, deux à la sortie). Il est ainsi possible de travailler avec une source étendue le long de l'axe du CMA (4 à $8 \mathrm{~mm}$, suivant les largeurs, réglables, définies pour chacune des fentes), tout en gardant une résolution acceptable (de l'ordre de $0,9 \%$ de l'énergie cinétique des électrons émis dans les expériences en question) et en conservant les propriétés de focalisation au premier ordre du CMA.

La partie laser de l'appareillage[49] comporte un laser pompe à gaz ionisé (argon ou krypton, noté Ar I dans la figure) pouvant produire jusqu'à 10 watts en monoraie et 20 watts en multiraies, un laser continu à colorant en anneau (DL I)et un système de miroirs et de lentilles qui transportent et focalisent le faisceau laser dans le volume source du CMA, dans lequel il pénètre à travers une fenêtre placée à l'angle de Brewster. La fréquence du laser est asservie sur la transition d'excitation voulue en utilisant la fluorescence produite par la désexcitation radiative du niveau optiquement excité dans un faisceau atomique auxiliaire et détectée à l'aide d'un photomultiplicateur (PMT I) précédé d'un filtre (F1). Le faisceau laser est perpendiculaire à l'axe du CMA afin de minimiser l'effet Doppler, le rayonnement synchrotron est colinéaire à l'axe du CMA afin de pouvoir mesurer des sections efficaces différentielles de photoionisation qui soient proportionnelles aux sections efficaces absolues, compte renu de la polarisation du rayonnement synchrotron. Cette section efficace différentielle a, en effet, pour expression, pour une cible non polarisée[50]:

avec:

$$
\mathrm{d} \sigma / \mathrm{d} \Omega=\sigma_{\mathrm{nl}} / 4 \pi\left(1-\beta_{\mathrm{n} l} / 2\left[\mathrm{P}_{2}(\cos \theta)-3 / 2\left(\cos 2 \omega \cos 2 \varphi \sin ^{2} \theta\right]\right.\right.
$$

$$
\begin{aligned}
& \mathrm{P}_{2}=\left(3 \cos ^{2} \theta-1\right) / 2 \\
& \omega=\text { ellipticité du rayonnement synchrotron }=\mathrm{tgb} / \mathrm{a} \text {, où b et a sont les petit et } \\
& \quad \text { grand axes de l'ellipse de polarisation } \\
& \theta=\text { angle entre les directions de propagation des photons et des photoélectrons } \\
& \varphi=\text { angle azimutal des photoélectrons, rapporté à l'axe principal de l'ellipse a. }
\end{aligned}
$$

On voit que, si les atomes cibles ne sont ni orientés, ni alignés (ce qui est le cas lorsqu'on travaille à des densités supérieures à $10^{11}$ atomes $/ \mathrm{cm}^{3}$ ), et si on collecte tous les photoélectrons émis dans le domaine $(0,2 \pi)$ de variation de l'angle $\varphi$, le choix de l'angle $\theta=$ $54^{\circ} 44^{\prime}$ (angle dit magique) entraîne la relation:

$$
\mathrm{d} \sigma_{\mathrm{n} l} / \mathrm{d} \Omega=\sigma_{\mathrm{nl}} / 4 \pi
$$

Dans la figure 1, on peut voir un deuxième système laser (ArII, DL II,...) permettant de transférer l'électron excité sur un deuxième niveau, d'énergie plus élevée, tel que cela est montré, pour le cas du sodium, dans le schéma d'excitation inséré dans la partie gauche de la figure. Ce deuxième système a été utilisé $[35,49,51]$ avec ACO en 1986-1987, mais il n'a pas encore été mis en oeuvre avec Super ACO.

Le très faible domaine d'énergie de photon à l'intérieur duquel les lasers à colorant continus sont accordables avec des puissances suffisantes (au moins $100 \mathrm{~mW}$ en sortie, de préférence plusieurs centaines de $\mathrm{mW}$ ) fait que seuls des atomes à faible potentiel d'ionisation ont pu, jusqu'à maintenant, être étudiés par cette méthode, c'est à dire les alcalins - Li, $\mathrm{Na}, \mathrm{K}$ et les alcalino-terreux - $\mathrm{Ca}, \mathrm{Ba}$.

Les transitions d'excitation d'un électron externe de ces atomes vers le premier niveau optiquement excitable sont les suivantes: 
Lithium: $\quad 1 s^{2} 2 s^{2} \mathrm{~S}_{1 / 2}+h v_{\text {laser }} \rightarrow 1 \mathrm{~s}^{2} 2 \mathrm{p}^{2} \mathrm{P}_{3 / 2}$ (ou ${ }^{2} \mathrm{P}_{1 / 2}$ ) à une longueur d'onde de $6708 \AA(1.85 \mathrm{eV})$, obtenue avec le colorant DCM.

Sodium: $\quad 2 s^{2} 2 p^{6} 3 s^{2} S_{1 / 2}+h v_{\text {laser }} \rightarrow 2 p^{6} 3 p^{2} P_{3 / 2}$ (ou ${ }^{2} P_{1 / 2}$ ) à une longueur d'onde de $5890 \AA(2,10 \mathrm{eV})$, obtenue avec la Rhodamine $6 \mathrm{G}$.

Potassium: $3 s^{2} 3 p^{6} 4 s^{2} S_{1 / 2}+h v_{\text {laser }} \rightarrow 3 p^{6} 4 p^{2} P_{3 / 2}$ (ou $2 P_{1 / 2}$ ) à une longueur d'onde de $7667 \AA(1,62 \mathrm{eV})$, obtenue avec le LD700.

Calcium : $\quad 3 s^{2} 3 p^{6} 4 s^{2}{ }^{1} S_{0}+h v_{\text {laser }} \rightarrow 3 s^{2} 3 p^{6} 4 s 4 p{ }^{1} P_{1}$ à une longueur d'onde de $4227 \AA(2,80 \mathrm{eV})$, obtenue avec le stilbène.

Barium: $\quad 5 s^{2} 5 p^{6} 6 s^{2}{ }^{1} s_{0}+h v_{\text {laser }} \rightarrow 5 p^{6} 6 s 6 p^{1} P_{1} \rightarrow 5 p^{6} 6 s 5 d{ }^{1,3} \mathrm{D}$ à une longueur d'onde de $5535 \AA(2,24 \mathrm{eV})$, obtenue avec la Rhodamine 110 .

Le dernier cas, celui du baryum, est spécial. L'existence des niveaux métastables ${ }^{1,3} \mathrm{D}$ à une énergie inférieure de $0,43 \mathrm{eV}$ à celle du premier niveau optiquement accessible $\left(6 \mathrm{~s} 6 \mathrm{p}{ }^{1} \mathrm{P}_{1}\right)$ fait que les atomes, initialement excités dans ce niveau, se désexcitent rapidement vers les niveaux métastables et que, dans de bonnes conditions expérimentales, pratiquement $100 \%$ des atomes de baryum peuvent être transférés dans ces états $1,3 \mathrm{D}$.

Pour les autres éléments, les densités relatives mesurées d'atomes portés dans le premier état excité varient, de quelques $\%$ dans le cas du calcium, à un maximum d'environ $30 \%$ dans le cas le plus favorable du sodium. Elles dépendent des conditions de polarisation du laser. L'expérience a aussi montré qu'elles dépendaient de façon critique de la densité d'atomes dans l'état fondamental: pour chaque élément, cette densité relative d'états excités passe par un maximum lorsqu'on fait croître la densité d'atomes dans l'état fondamental, puis se met à décroître. Ceci est le résultat de deux facteurs antagonistes: le piégeage de radiation[52] et la dépopulation par collision. Les atomes excités se désexcitent vers l'état fondamental en émettant un rayonnement de fluorescence qui est aussitôt absorbé par les atomes voisins, portés ainsi, à leur tour, dans l'état excité. Ce processus peut se produire plusieurs dizaines de fois avant que le rayonnement échappe du volume source du CMA. La polarisation du rayonnement de fluorescence émis dans la désexcitation des atomes excités a été mesurée quantitativement $[53,54]$ dans les cas du lithium et du sodium. A cause de ce piégeage, tout effet d'orientation ou d'alignement induit par l'excitation sélective des différents niveaux magnétiques est détruit pour des valeurs des densités d'atomes dans l'état fondamental supérieures à $10^{11}$ atomes $/ \mathrm{cm}^{3}$. Lorsqu'on continue à faire croître cette densité après avoir atteint le maximum de densité des atomes dans l'êtat excité, celle-ci décroît, les atomes étant alors désexcités par collision. Cet effet a été quantitativement mesuré dans le cas du baryum[55].

Les premiers spectres de photoélectrons résultant de l'excitation ou de l'ionisation d'électrons de couches internes par le rayonnement synchrotron dans un atome excité par laser ont été obtenus dès 1981 pour le sodium[ $[1,42,43]$, et 1982 pour le baryum[ $[43,44,56]$. La figure 2 montre l'existence, dans l'atome excité de sodium, d'une excitation résonante du type $2 p^{6} 3 p^{2} P_{3 / 2} \rightarrow 2 p^{5} 3 s 3 p{ }^{2} D_{3 / 2}$ et ${ }^{2} D_{5 / 2}$, suivie de l'autoionisation de ces états doublement excités dans le continuum $2 \mathrm{p}^{6}{ }^{1} \mathrm{~S}_{\mathrm{o}}$ de l'ion $\mathrm{Na}^{+}$. Le processus de photoionisation résonante se traduit pratiquement par l'augmentation locale de la section efficace de photoionisation de l'électron excité 3p. C'est ce qui est observé dans le spectre de la partie basse de la figure 2: en présence du laser accordé à l'énergie de la transition $3 \mathrm{~s} \rightarrow 3 \mathrm{p}$, on voit apparaître une raie d'électrons intense à l'énergie de liaison de l'électron $3 \mathrm{p}(3,03 \mathrm{eV})$. Dans ces premières expériences[1,42], le bruit de fond était élevé à cause de la détection d'électrons de basse énergie produits dans des collisions d'ionisation associative et d'ionisation Penning entre atomes excités[57]. De plus, la densité relative d'atomes excités ne dépassait guère $10 \%$. L'optimisation des conditions expérimentales permit d'obtenir rapidement des densités voisines 


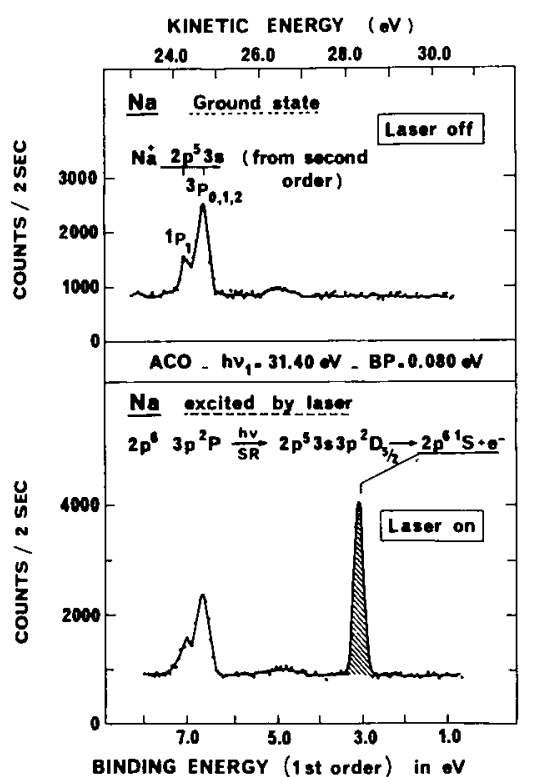

Fig. 2 - Spectres d'électrons produits par lirradiation, avec des photons de $31,40 \mathrm{eV}$ et $62,80 \mathrm{eV}$, du sodium atomique dans son état fondamental (raies autour d'une énergie cinétique de $25 \mathrm{eV}$ ) et dans l'état excité $3 p$ (raies à 3,03 eV d'énergie de liaison) (Bizau et al., 1981, réf. I).

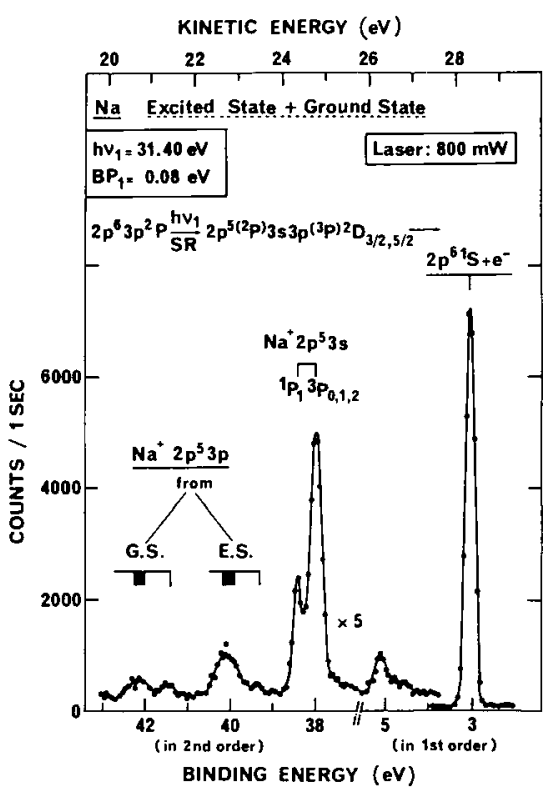

Fig. 3 - Spectre d'électrons enregistré aux mêmes énergies de photons que dans le bas de la figure 2 , mais dans des conditions expérimentales améliorées. De plus, on observe aussi ici la photoionisation directe de l'état excité vers $40 \mathrm{eV}$ d'énergie de liaison (Wuilleumier, 1982, réf. 43).

de $30 \%$ et de réduire sensiblement le bruit de fond, comme le démontre la figure 3 enregistrée à la même énergie de photons, dès la seconde série d'expériences[2, 43].

La source émettrice de photons a été, pendant sept ans, jusqu'en 1988, l'anneau ACO auquel a succédé l'anneau de stockage de la troisième génération Super ACO, construit à Orsay et utilisé depuis 1989. A partir de 1985, Jes expériences du même type ont commencé[58] à Berlin, avec l'anneau BESSY et un montage d'abord assez semblable à celui de la figure 1.

L'augmentation significative du flux de photons disponible sur les aimants de déviation de Super ACO, par rapport à ceux d'ACO, ainsi que la possibilité, pour les chercheurs travaillant à BESSY, d'utiliser un onduleur ont permis de mettre en oeuvre de nouveaux dispositifs afin d'analyser angulairement les spectres d'électrons émis[39, 59, 60, 64]. Nous montrons, dans la figure 4 , le dispositif mis au point à Orsay $[59,60]$ qui permet d'enregistrer simultanément jusqu'à neuf spectres d'électrons émis à différentes valeurs de l'angle $\varphi$. La figure montre une couronne de six channeltrons placée perpendiculairement à l'axe du CMA entre les deux cylindres, sur le cercle de focalisation, et qui a été utilisée pour les premières mesures d'un paramètre de distribution angulaire dans la photoionisation directe d'électrons de couche interne dans un atome excité. A la différence d'autres montages dans lesquels le spectromètre à électrons doit tourner, rien n' est mobile dans le système choisi à Orsay et, facteur essentiel, tous les spectres d'électrons sont détectés en même temps: ceci permet de 


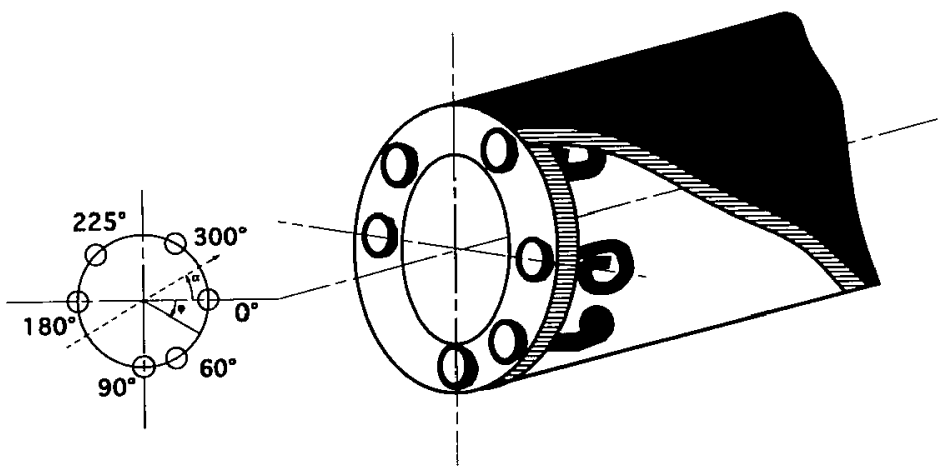

Fig. 4 - Système de détection à six channeltrons pour l'étude des distributions angulaires

s'affranchir de l'effet des variations lentes de densité des atomes aussi bien dans l'état fondamental que dans l'état excité. Toute modification de la densité d'atomes dans l'état excité se traduit, de plus, par un déplacement de l'échelle apparente des énergies cinétiques, à cause de la variation immédiate du potentiel plasma créé par les collisions ionisantes, potentiel qui peut facilement atteindre 1 volt. Dans ces conditions, il est toujours difficile de compter longtemps un spectre d'électrons provenant de la photoionisation d'un atome excité, à cause des instabilités du laser à colorant fonctionnant toujours à pleine puissance dans un environnement plutôt défavorable. Les intensités d'électrons étant diminuées d'environ un ordre de grandeur dans la configuration du système permettant l'analyse angulaire, il était très important que les variations éventuelles des conditions expérimentales soient strictement les mêmes pour toutes les voies correspondant à différentes valeurs de l'angle $\varphi$.

\section{RESULTATS OBTENUS}

Nous ne reviendrons pas ici en détail sur l'intérêt général que présente cette nouvelle technique pour la physique atomique, ces questions ayant été largement discutées dans des revues précédentes $[45,86]$. Nous nous contenterons ici de donner un résumé des expériences faites et de présenter quelques résultats particulièrement intéressants.

Compte tenu des intensités relativement faibles des flux de photons disponibles il y a plus de dix ans, seuls les processus résonants, dont la section efficace est renforcée par l'existence d'un état discret dégénéré avec un continuum d'ionisation, ont pu être étudiés dans un premier temps, à l'exception du cas particulier du baryum. L'utilisation des nouvelles sources plus intenses de rayonnement synchrotron a permis, d'une part, de mesurer, dans des atomes excités, des sections efficaces partielles[37, 61-63] de photoionisation directe en couche interne, et tout récemment, d'étudier la distribution angulaire $[59,60,64]$ de photoélectrons émis, et, d'autre part, d'aborder, avec des densités d'atomes excités suffisamment faibles, l'étude des effets d'alignement induits par l'excitation laser sur la photoexcitation d'électrons de couche interne $[39,54]$. Nous diviserons ce paragraphe en deux parties distinctes, consacrées respectivement aux processus d'autoionisation et aux processus de photoionisation directe dans le continuum.

\section{Processus résonants}

La Table II donne le résumé de l'ensemble des expériences ayant porté sur la photoionisation résonante d'atomes excités, c'est à dire sur l'excitation d'électrons internes sur une orbitale excitée, peuplant un état autoionisant de l'atome qui se relaxe en des temps très courts $\left(\approx 10^{-13}\right.$ à $10^{-14}$ seconde). Nous avons déja vu, dans les figures 2 et 3 , un exemple 
Table II. Processus de photoionisation résonante dans des atomes excités étudiés par utilisation combinée de rayonnements laser(s) et synchrotron.

\begin{tabular}{|c|c|c|c|c|c|}
\hline $\begin{array}{l}\text { Atome et état } \\
\text { initial }\end{array}$ & $\begin{array}{c}\text { Etat atomique } \\
\text { optiquement } \\
\text { excité }\end{array}$ & $\begin{array}{c}\text { Configuration } \\
\text { de l'état excité } \\
\text { par RS }\end{array}$ & $\begin{array}{l}\text { Etat ionique } \\
\text { final }\end{array}$ & $\begin{array}{l}\text { Grandeurs } \\
\text { mesurées }\end{array}$ & Références \\
\hline $\begin{array}{c}\mathbf{N a} \\
2 p^{6} 3 s^{2} S_{1 / 2}\end{array}$ & $2 p^{6} 3 p^{2} P_{3 / 2}$ & $2 p^{5} 3 s 3 p$ & $\begin{array}{c}\mathrm{Na}^{+} \\
2 \mathrm{p}^{61} \mathrm{~S}_{\mathrm{o}}\end{array}$ & $\begin{array}{l}\text { Expérience de } \\
\text { faisabilité }\end{array}$ & $\begin{array}{c}1981 \\
\text { Bizau et al, } \\
\text { réfs. } 1 \text { et } 42 \\
\text { Wuilleumier, } \\
\text { réf. } 43 \\
\end{array}$ \\
\hline $\begin{array}{c}\mathbf{N a} \\
2 \mathrm{p}^{6} 3 \mathrm{~s}^{2} \mathrm{~S}_{1 / 2}\end{array}$ & $2 p^{6} 3 p^{2} P_{3 / 2}$ & $2 p^{5} 3 s 3 p$ & $\begin{array}{c}\mathrm{Na}^{+} \\
2 \mathrm{p}^{6}{ }^{1} \mathrm{~S}_{\mathrm{o}}\end{array}$ & $\begin{array}{l}\text { - Energies } \\
\text { - Forces d'os- } \\
\text { cillateur }\end{array}$ & $\begin{array}{c}1984-1985 \\
\text { Wuilleumier } \\
\text { réf. } 44 \\
\text { Bizau et al., } \\
\text { réf. } 34\end{array}$ \\
\hline $\begin{array}{c}\mathbf{N a} \\
2 \mathrm{p}^{6} 3 \mathrm{~s}^{2} \mathrm{~S}_{1 / 2}\end{array}$ & $\begin{array}{c}2 \mathrm{p}^{6} 3 \mathrm{p}^{2} \mathrm{P}_{1 / 2} \\
2 \mathrm{P}_{3 / 2}\end{array}$ & $2 p^{5} 3 s 3 p$ & $\begin{array}{c}\mathrm{Na}^{+} \\
2 \mathrm{p}^{6}{ }^{1} \mathrm{~S}_{\mathrm{o}}\end{array}$ & $\begin{array}{l}\text { - Energies } \\
\text { - Forces d'os- } \\
\text { cillateur } \\
\text { - Influence } \\
\text { polarisation }\end{array}$ & $\begin{array}{c}1987 \\
\text { Meyer et al., } \\
\text { refs. } 53 \text { et } 68 \\
\text { Prescher, réf. } \\
\quad 65\end{array}$ \\
\hline $\begin{array}{c}\mathrm{Na} \\
2 \mathrm{p}^{6} 3 \mathrm{~s}^{2} \mathrm{~S}_{1 / 2}\end{array}$ & $\begin{array}{l}2 p^{6} 4 d^{2} D_{5 / 2} \\
2 p^{6} 5 s^{2} S_{1 / 2} \\
2 p^{6} 4 p^{2} P_{3 / 2} \\
2 p^{6} 4 s^{2} S_{1 / 2}\end{array}$ & $\begin{array}{l}2 p^{5} 3 s 4 d \\
2 p^{5} 3 s 5 s \\
2 p^{5} 3 s 4 p \\
2 p^{5} 3 s 4 s\end{array}$ & $\begin{array}{c}\mathrm{Na}^{+} \\
2 \mathrm{p}^{6}{ }^{1} \text { So }\end{array}$ & $\begin{array}{l}\text { - Energies } \\
\text { - Forces d'os- } \\
\text { cillateur }\end{array}$ & $\begin{array}{l}1986-1987 \\
\text { Ferray et al., } \\
\text { réf. } 35 \\
\text { Cubaynes et } \\
\text { al., réf. } 51 \\
\text { Carré et al., } \\
\text { réf. } 49\end{array}$ \\
\hline $\begin{array}{c}\text { Ba } \\
5 p^{6} 6 s^{2}{ }^{1} S_{0}\end{array}$ & $\begin{array}{l}5 p^{6} 6 s_{6} p^{1} P_{1} \\
5 p^{6} 6 s 5 d \\
{ }^{2} D_{3 / 2,5 / 2} \\
\end{array}$ & $\begin{array}{l}5 p^{5} 6 s 6 p n l \\
5 p^{5} 6 s 5 d n^{\prime} l^{1}\end{array}$ & \begin{tabular}{r}
\multicolumn{2}{c}{$\mathrm{Ba}^{+}$} \\
$5 \mathrm{p}^{6} 6 \mathrm{~s}^{2} \mathrm{~S}_{1 / 2}$ \\
$5 \mathrm{p}^{6} 5 \mathrm{~d}^{2} \mathrm{D}_{3 / 2}$ \\
${ }^{2} \mathrm{D}_{5 / 2}$ \\
\end{tabular} & $\begin{array}{l}\text { Expérience de } \\
\text { faisabilité }\end{array}$ & $\begin{array}{c}1985 \\
\text { Nunnemann } \\
\text { et al., réf. } 58\end{array}$ \\
\hline $\begin{array}{c}\mathbf{B a} \\
4 \mathrm{~d}^{10} 5 \mathrm{~s}^{2} 5 \mathrm{p}^{6} \\
6 \mathrm{~s}^{2}{ }^{1} \mathrm{~S}_{\mathrm{o}}\end{array}$ & $\begin{array}{c}4 d^{10} 5 s^{2} 5 p^{6} \\
6 s 5 d^{1} P_{1}\end{array}$ & $\begin{array}{c}4 \mathrm{~d}^{10} 5 \mathrm{~s}^{2} 5 \mathrm{p}^{5} \\
n \ln \mathrm{l}^{\prime} n^{\prime \prime l} l^{\prime \prime} \\
4 \mathrm{~d}^{9} 5 \mathrm{~s}^{2} 5 \mathrm{p}^{6} 6 \mathrm{~s} \\
n \ln ^{\prime} \mathrm{l}^{\prime \prime} \mathrm{l}^{\prime \prime}\end{array}$ & $\begin{array}{l}\mathrm{Ba}^{+} \\
4 \mathrm{~d}^{10} 5 \mathrm{~s}^{2} 5 \mathrm{p}^{6} 6 \mathrm{~s} \\
5 \mathrm{~d} \\
4 \mathrm{~d}^{10} 5 \mathrm{~s}^{2} 5 \mathrm{p}^{5} 6 \mathrm{~s} \\
5 \mathrm{~d} \\
\end{array}$ & $\begin{array}{l}\text { - Energies } \\
\text { - Fonctions } \\
\text { d'excitation }\end{array}$ & $\begin{array}{c}\text { 1987-1989 } \\
\text { Bizau et al., } \\
\text { réf. } 55,69\end{array}$ \\
\hline $\begin{array}{c}\text { Ca } \\
3 p^{6} 4 s^{2}{ }^{1} S_{0}\end{array}$ & $3 p^{6} 4 s 4 p{ }^{1} P_{1}$ & $3 \mathrm{p}^{5} 3 \mathrm{dn} \ln \mathrm{l}^{\prime}$ & $\begin{array}{c}3 \mathrm{p}^{6} 4 \mathrm{~s}^{2} \mathrm{~S}_{1 / 2} \\
4 \mathrm{p} \\
3 \mathrm{~d}\end{array}$ & $\begin{array}{l}\text { - Energies } \\
\text { - Fonction } \\
\text { d'excitation }\end{array}$ & $\begin{array}{c}1987-1992 \\
\text { Meyer et al., } \\
\text { réf. } 70 \\
\text { Baier et al., } \\
\text { ref. } 89 \\
\end{array}$ \\
\hline $\begin{array}{c}\mathbf{L i} \\
1 \mathrm{~s}^{2} 2 \mathrm{~s}^{2} \mathrm{~S}_{1 / 2}\end{array}$ & $\begin{array}{c}1 \mathrm{~s}^{2} 2 \mathrm{p}^{2} \mathrm{P}_{1 / 2} \\
2 \mathrm{P}_{3 / 2} \\
\text { atomes } \\
\text { alignés }\end{array}$ & $1 \mathrm{~s} 2 \mathrm{snl}$ & $\begin{array}{c}\mathrm{Li}^{+} \\
1 \mathrm{~s}^{2}{ }^{1} \mathrm{~S}_{\mathrm{O}}\end{array}$ & $\begin{array}{l}\text { - Energies } \\
\text { - Fonctions } \\
\text { d'excitation } \\
\text { - Distribution } \\
\text { angulaire }\end{array}$ & $\begin{array}{c}1987-1992 \\
\text { Meyer et al., } \\
\text { réf. } 38 \\
\text { Pahler et al. } \\
\text { réfs. } 39 \text { et } 54\end{array}$ \\
\hline
\end{tabular}


particulièrement simple dans le cas de l'excitation d'un électron $2 \mathrm{p}$ dans un atome de sodium préparé dans l'état $2 \mathrm{p}^{6} 3 \mathrm{p}^{2} \mathrm{P}_{3 / 2}$. Après la mise en évidence du phénomène, les études poursuivies ont permis de mesurer les forces d'oscillateur des transitions $2 p^{6} 3 p \rightarrow 2 p^{5} 3 s 3 p$ [34, 44]. Des mesures ultérieures[65] ont fourni des résultats sensiblement analogues à partir de l'état excité $2 \mathrm{p}^{6} 3 \mathrm{p}^{2} \mathrm{P}_{1 / 2}$.

Après la réussite des expériences de faisabilité sur le baryum $[2,44,56]$, il a été possible de mesurer les énergies d'un certain nombre de transitions d'excitation d'un électron interne $5 p$ dans les états $5 p^{6} 5 \mathrm{~s} 5 \mathrm{~d}{ }^{1,3} \mathrm{D}$ et de déterminer les fonctions d'excitation, proportionnelles aux sections efficaces, pour différents états finals dans le continuum[66,67]. La figure 5 montre un exemple particulièrement spectaculaire dans le cas de la photoionisation du baryum par des photons de $19,71 \mathrm{eV}$. A cette énergie d'excitation résonante, on remarque (partie haute de la figure) que, dans la photoionisation de l'état fondamental, les raies satellites (états finals $5 \mathrm{p}^{6} 5 \mathrm{~d}$, $5 \mathrm{p}^{6} 7 \mathrm{~s}$ notamment) sont présentes avec une intensité importante. Dans le spectre de l'état excité (partie basse de la figure), toutes les raies provenant de la photoionisation de l'état fondamental ont pratiquement disparues, signe que la quasi totalité des atomes a été transférée dans les états excités $6 s 5 \mathrm{~d} 1,3 \mathrm{D}$. De nombreuses autres raies (raie principale et raies satellites), issues de l'atome excité, apparaissent. Lorsque les niveaux autoionisants sont suffisamment séparés, les raies émises dans la photoionisation de l'état ${ }^{1} \mathrm{D}$ et celles émises dans la photoionisation de l'état ${ }^{3} \mathrm{D}$ peuvent être résolues.

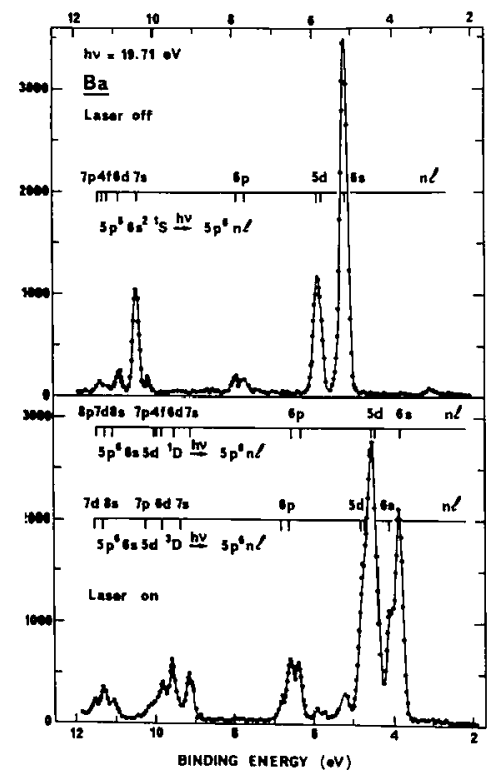

Fig. 5 - Spectres d'électrons du baryum à une énergie de photons de 19,71 eV. Dans la partie supérieure, les atomes sont dans l'état fondamental; dans la partie inférieure, plus de $90 \%$ des atomes ont été transférés dans les états excités $5 p^{6} 6 s 5 d$ $1,3 \mathrm{D}$ (voir réduction des raies $6 s$ et $5 d$ de l'état fondamental) (Cubaynes et al., 1986, réf. 66).

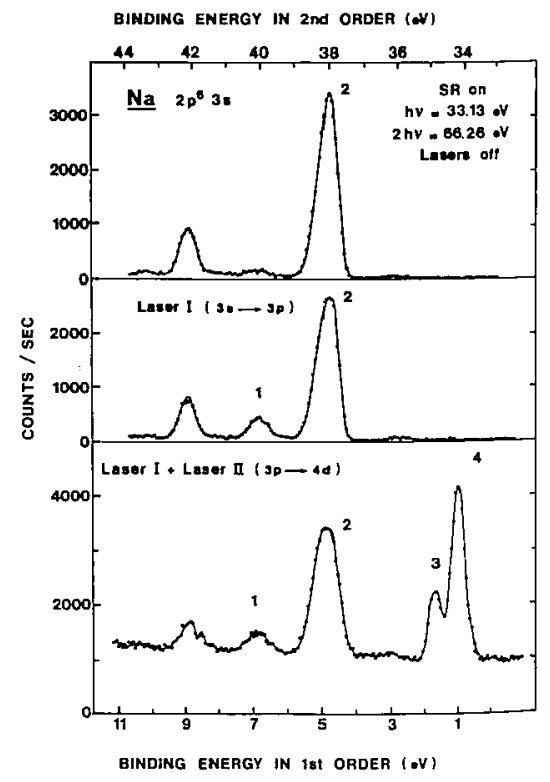

Fig. 6 - Spectres d'électrons mesurés dans le sodium avec tes photons de $33,13 \mathrm{eV}$ (raies notées 3 et 4 , dues à la photoionisation résonante d'électrons $4 p$ dans l'état $2 p^{6} 4 p^{2} P_{3 / 2}$ et $4 d$ dans l'état

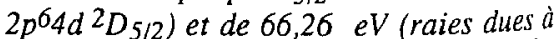
la photoionisation directe d'un électron $2 p$ dans les états excité $3 p$, raie 1 , et fondamental, raie 2) (Carré et al., 1990 , réf. 59). 
Nous illustrons également, dans la figure 6, les expériences ayant permis d'explorer avec deux lasers utilisés en cascade $[35,49,51]$, les processus de photoexcitation d'un électron $2 p$ dans des atomes de sodium portés dans des états très excités tels que $2 p^{6} 4 d$ et $2 p^{6} 4 p$ : on peut distinguer les raies de photoélectrons correspondant à la photoionisation directe d'un électron $2 \mathrm{p}$ dans l'état excité $3 \mathrm{p}^{2} \mathrm{P}_{3 / 2}$ (pic noté 1 ) et dans l'état fondamental (pic noté 2 ) ainsi que ceux dus à la photoionisation résonante de l'électron $4 \mathrm{p}$ à partir de l'état excité $2 \mathrm{p}^{6} 4 \mathrm{p}^{2} \mathrm{P}_{3 / 2}$ (pic noté 3 ) et de l'électron $4 d$ à partir de l'état excité $2 \mathrm{p}^{64} \mathrm{~d}^{2} \mathrm{D}_{5 / 2}$ (pic noté 4 ), tous ces états étant présents simultanément dans la vapeur irradiée par le rayonnement synchrotron.

Pour en terminer avec ces quelques exemples de processus résonants, nous montrons dans les figures 7 et 8 , les premiers résultats obtenus dans l'étude des processus d'autoionisation dans des atomes de lithium excités et alignés par laser dans les états $1 \mathrm{~s}^{2} 2 \mathrm{p}$ ${ }^{2} \mathrm{P}_{1 / 2}$ et ${ }^{2} \mathrm{P}_{3 / 2}$. La figure 7 donne directement la fonction d'excitation pour la photoionisation

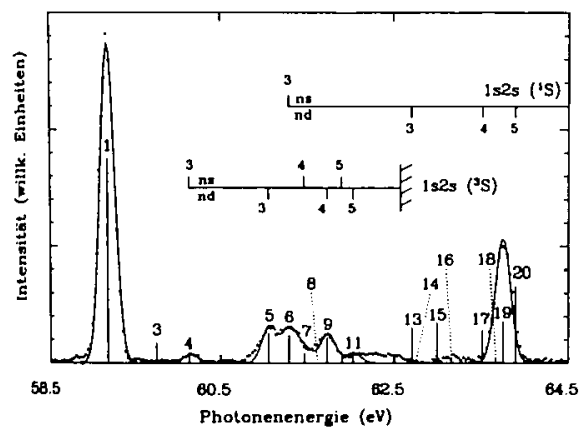

Fig. 7 - Fonction d'excitation pour lionisation résonante de l'électron $2 p$ dans l'état excité $1 s^{2} 2 p{ }^{2} P_{1 / 2}$ du lithium en fonction de l'énergie des photons (Prescher, 1988, réf. 65 et Pahler, 1992, réf. 54).

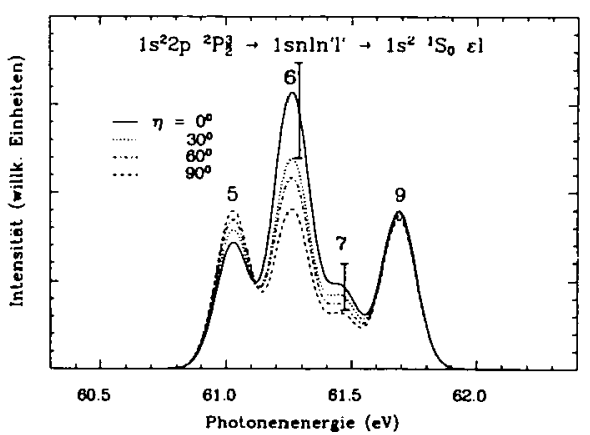

Fig. 8 - Variation de l'intensité relative des raies 5 a 9 de la figure 7 , mais pour l'état excité ${ }^{2} P_{3 / 2}$, d̀ plusieurs valeurs del'angle $\eta$ entre les deux axes de polarisation (Pahler, 1992, réf. 54).

résonante d'un électron $2 \mathrm{p}$ dans l'atome de lithium excité dans l'état ${ }^{2} \mathrm{P}_{1 / 2}$, suivant le schéma: ${ }_{1 s^{2} 2 \mathrm{p}}{ }^{2} \mathrm{P}_{1 / 2} \rightarrow 1$ sĩin'l' $\rightarrow 1 \mathrm{~s}^{2}{ }^{1} \mathrm{~S}_{\mathrm{o}}+\varepsilon \mathrm{l}$, car cet état n'est pas susceptible d'être aligné par le laser. Par contre, dans la figure 8 , obtenue en partant de l'état excité $1 \mathrm{~s}^{2} 2 \mathrm{p}{ }^{2} \mathrm{P}_{3 / 2}$, l'intensité relative des raies 5 à 9 de la figure 7 est représentée pour différentes valeurs de l'angle $\eta$ entre les deux axes de polarisation du laser et du rayonnement synchrotron. Cette figure démontre que la variation de cette intensité dépend fortement de la symétrie de l'état autoionisant, de l'alignement de l'état optiquement excité par le laser et de l'orientation reláive des axes de polarisation des deux rayonnements. Les résultats montrés dans cette figure furent expliqués par l'analyse de la distribution angulaire des électrons émis en fonction de leur angle d'émission $[39,54]$.

\section{Processus de photoionisation directe; sections efficaces partielles de photoionisation}

Nous donnons, dans la Table III, la liste de toutes les études qui ont porté sur la photoionisation directe dans le continuum en couches internes d'atomes optiquement excités et qui ont été publiées à ce jour. Dans la fin de ce chapitre, nous illustrerons quelques uns des résultats les plus importants obtenus. 
Table III. Processus de photoionisation directe dans le continuum étudiés par utilisation combinée de rayonnements laser et synchrotron

\begin{tabular}{|c|c|c|c|c|}
\hline$\frac{\text { Elément et état }}{\frac{\text { atomique }}{\text { excité }}}$ & $\frac{\text { Processus }}{\text { étudié }}$ & $\frac{\text { Configuration }}{\text { de l'état final }}$ & $\frac{\text { Grandeurs }}{\underline{\text { mesurées }}}$ & Références \\
\hline $\begin{array}{c}\mathrm{Na} \\
1 \mathrm{~s}^{2} 2 \mathrm{p}^{6} \mathrm{p}^{2} \mathrm{P}_{3 / 2}\end{array}$ & $\begin{array}{l}\text { Photoionisation } \\
\text { simple de l'é- } \\
\text { lectron } 2 p \text { (raie } \\
\text { principale) }\end{array}$ & $2 \mathrm{p}^{5} 3 \mathrm{p}+\varepsilon s, \varepsilon d$ & $\begin{array}{l}\text { Expérience de faisabilité } \\
\text { (observation du spectre } \\
\text { de photoélectrons) }\end{array}$ & $\begin{array}{l}\quad 1981 \\
\text { Bizau et al., } \\
\text { réfs. } 1 \text { et } 42 \\
\text { Wuilleumier } \\
\text { réf. } 43\end{array}$ \\
\hline $\begin{array}{c}\text { Ba } \\
4 d^{10} 5 s^{2} 5 p^{6} 6 s \\
5 d^{1,3} D\end{array}$ & $\begin{array}{c}\text { Photoionisation } \\
\text { simple en sous } \\
\text { couches } \\
\text { 4d,5s,5p,6s,5d } \\
\text { (raies } \\
\text { principales) }\end{array}$ & $\begin{array}{c}4 d^{9} 5 s^{2} 5 p^{6} 6 s 5 d \\
+\varepsilon f, \varepsilon p \\
5 s 5 p^{6} 6 s 5 d+\varepsilon p \\
5 p^{5} 6 s 5 d+\varepsilon s, \varepsilon d \\
5 p^{6} 6 s+\varepsilon f, \varepsilon p \\
5 p^{6} 5 d+e p\end{array}$ & $\begin{array}{c}\text { Expériences de } \\
\text { faisabilité (observa- } \\
\text { tion des spectres de } \\
\text { photoélectrons) }\end{array}$ & $\begin{array}{l}1982 \\
\text { Wuilleumier } \\
\text { réf. } 2 \\
\text { Bizau et al., } \\
\text { réf. } 56\end{array}$ \\
\hline $\begin{array}{c}\text { Ba } \\
5 s^{2} 5 \mathrm{p}^{6} 6 \mathrm{~s} 5 \mathrm{~d} \\
1,3 \mathrm{D}\end{array}$ & $\begin{array}{l}\text { Photoionisation } \\
\text { en couche } 5 \mathrm{~d}\end{array}$ & $5 p^{6} 6 s+\varepsilon f, \varepsilon p$ & $\begin{array}{l}\text { Section efficace de } \\
\text { photoionisation en } \\
\quad \text { couche 5d }\end{array}$ & $\begin{array}{l}1986 \\
\text { Bizau et al., } \\
\text { réf. } 36\end{array}$ \\
\hline $\begin{array}{c}\mathbf{B a} \\
5 \mathrm{~s}^{2} 5 \mathrm{p}^{6} 6 \mathrm{~s} 5 \mathrm{~d} \\
1,3 \mathrm{D}\end{array}$ & $\begin{array}{l}\text { Photoionisation } \\
\text { en sous- } \\
\text { couches } 5 \text { s et } \\
5 p\end{array}$ & $\begin{array}{c}5 s 5 p^{6} 6 s 5 d+\varepsilon p \\
5 s^{2} 5 p^{5} 6 s 5 d+ \\
\varepsilon f, \varepsilon p\end{array}$ & $\begin{array}{c}\text { Sections efficaces de } \\
\text { photoionisation simple en } \\
\text { sous-couches 5s,5p } \\
\text { et } 6 \mathrm{~s}\end{array}$ & $\begin{array}{l}1987 \\
\text { Bizau et al., } \\
\text { réf. } 69\end{array}$ \\
\hline $\begin{array}{c}\mathbf{N a} \\
2 \mathrm{p}^{6} 3 \mathrm{p}^{2} \mathrm{P}_{3 / 2}\end{array}$ & $\begin{array}{l}\text { Photoionisation } \\
\text { en sous-couche } \\
2 \mathrm{p} \text {, simple et } \\
\text { accompagnée } \\
\text { d'excitation }\end{array}$ & $\begin{array}{l}2 p^{5} 3 p+\varepsilon d, \varepsilon s \\
2 p^{5} 4 p+\varepsilon d, \varepsilon s \\
2 p^{5} 3 d \text { ou } 2 p^{5} 4 s \\
\quad+\varepsilon d, \varepsilon s\end{array}$ & $\begin{array}{l}\text { - Intensité relative des } \\
\text { satellites de corrélation } \\
\text { - Sections efficaces de } \\
\text { photoionisation en } \\
\text { couche } 2 \text { p (raies } \\
\text { principale et satellites) }\end{array}$ & $\begin{array}{l}1989 \\
\text { Cubaynes et } \\
\text { al., réfs. } 37 \\
\quad \text { et } 87\end{array}$ \\
\hline $\begin{array}{c}\mathbf{N a} \\
2 \mathrm{~s}^{2} 2 \mathrm{p}^{6} 3 \mathrm{p}^{2} \mathrm{P}_{3 / 2}\end{array}$ & $\begin{array}{l}\text { Photoionisation } \\
\text { en sous-couche } \\
2 \text { s, simple et } \\
\text { accompagnée } \\
\text { d'excitation }\end{array}$ & $\begin{array}{c}2 s 2 p^{6} 3 p+\varepsilon p \\
2 s^{6} p^{6} 4 p+\varepsilon p \\
2 s 2 p^{6} 3 d, 4 s+\varepsilon p\end{array}$ & $\begin{array}{l}\text { - Intensité relative des } \\
\text { satellites de corrélation } \\
\text { - Sections efficaces de } \\
\text { photoionisation en } \\
\text { couche } 2 s \text { (raies } \\
\text { principale et satellites) }\end{array}$ & $\begin{array}{l}1990 \\
\text { Richter et } \\
\text { al., réf. } 61\end{array}$ \\
\hline
\end{tabular}




\begin{tabular}{|c|c|c|c|c|}
\hline $\begin{array}{c}\mathbf{K} \\
3 \mathrm{~s}^{2} 3 \mathrm{p}^{64 \mathrm{p}^{2} \mathrm{P}_{3 / 2}}\end{array}$ & $\begin{array}{l}\text { Photoionisation } \\
\text { en sous-couche } \\
\text { 3p, simple et } \\
\text { accompagnée } \\
\text { d'excitation }\end{array}$ & $\begin{array}{c}3 p^{5} 4 p+\varepsilon d, \varepsilon s \\
3 p^{5} 5 p+\varepsilon d, \varepsilon s \\
3 p^{5} 4 s, 4 d+\varepsilon d, \\
\varepsilon s\end{array}$ & $\begin{array}{c}\text { Intensité relative des } \\
\text { satellites de corrélation } \\
\\
\text { Sections efficaces de } \\
\text { photoionisation en sous- } \\
\text { couche } 3 p \text { (raies } \\
\text { principale etsatellites) }\end{array}$ & $\begin{array}{l}1991 \\
\text { Wuilleumier } \\
\text { et al., réf. } 62 \\
\text { Cubaynes et } \\
\text { al. , réf. } 63\end{array}$ \\
\hline $\begin{array}{c}\mathbf{N a} \\
2 \mathrm{p}^{63} \mathrm{p}^{2} \mathrm{P}_{3 / 2}\end{array}$ & $\begin{array}{l}\text { Photoionisation } \\
\text { en sous-couche } \\
2 p, \text { simple et } \\
\text { accompagnée } \\
\text { d'excitation }\end{array}$ & $\begin{array}{c}2 p^{5} 3 p+\varepsilon d, \varepsilon s \\
2 p^{5} 4 p+\varepsilon d, \varepsilon s \\
2 p^{5} 3 d, 4 s+\varepsilon d, \\
\varepsilon s\end{array}$ & $\begin{array}{l}\text { Dépendance angulaire } \\
\text { de l'intensité relative des } \\
\text { satellites de corrélation }\end{array}$ & $\begin{array}{c}1992 \\
\text { Cubaynes et } \\
\text { al., réfs. } 60 \mathrm{et} \\
64\end{array}$ \\
\hline $\begin{array}{c}\mathrm{Na} \\
2 \mathrm{p}^{63} \mathrm{p}^{2 \mathrm{P}_{3 / 2}}\end{array}$ & $\begin{array}{l}\text { Photoionisation } \\
\text { simple en sous- } \\
\text { couche } 2 p\end{array}$ & $2 \mathrm{p}^{5} 3 \mathrm{p}+\varepsilon d, \varepsilon s$ & $\begin{array}{l}\text { Mesure du paramètre de } \\
\text { distribution angulaire } \beta \\
\text { de l'électron éjecté de la } \\
\text { sous-couche } 2 \mathrm{p}\end{array}$ & $\begin{array}{l}1992 \\
\text { Bizau et al. } \\
\text { réf. } 82 \text { et } \\
\text { Rouvellou et } \\
\text { al., réf. } 84\end{array}$ \\
\hline
\end{tabular}

Rappelons d'abord brièvement la nature des processus étudiés par la spectrométrie d'électrons. Dans le processus de photoionisation, les orbitales électroniques occupées par les électrons restant dans l'ion résiduel après le départ de l'électron éjecté dans le continuum peuvent être "apparemment" les mêmes que dans l'état initial, bien qu'ayant subi le phénomène de relaxation. Elles peuvent aussi, au moins pour l'une d'entre elles, être différentes, un peu de l'énergie disponible du photon incident ayant pu être utilisée pour transférer un deuxième électron, appartenant à la même couche ou à une couche plus externe, sur une orbitale d'énergie plus élevée. Dans un spectre de photoélectrons, il correspond respectivement deux groupes de raies distinctes à chacun de ces processus; la raie dite "principale", qui présente parfois une stucture fine due, par exemple, à l'interaction spin-orbite, et les raies dites satellites, situées dans une région d'énergie cinétique plus faible que celle de la raie principale, qui sont la manifestation explicite des effets de corrélation, puisque leur existence est interdite dans le modèle de l'atome à un électron.

Le deuxième électron peut changer son nombre quantique principal et/ou son nombre quantique orbital: lorsque ce dernier est le même que dans l'état initial, l'effet est principalement attribuable à la relaxation du coeur et est appelé effet "shake up" dans le modèle de l'approximation soudaine; lorsqu'il a changé d'une unité, il faut introduire l'interaction avec l'électron éjecté dans le continuum pour expliquer l'échange de moment orbital. L'origine de ce deuxième processus a reçu plusieurs noms, suivant les écoles théoriques qui l'ont étudié, tels que diffusion interne de l'électron ("internal scattering")[71] éjecté, l'excitation du deuxième êlectron étant attribuée à une collision à l'intérieur de l'atome de cet électron avec l'un des électrons restant, interaction de configuration dans l'état final du continuum (CSCI = "Continuum State Configuration Interaction"[72]); plus empiriquement, cet effet a souvent été désigné par les expérimentateurs sous le nom de "shake up conjugué"[73], pour le distinguer de l'effet shake up proprement dit. Une partie de l'intensité des satellites shake up produits sans changement du nombre quantique orbital a aussi été interprété dans le formalisme de l'interaction de configuration (FISCI = "Final Ionic State Configuration interaction"; ISCI = Initial State Configuration Interaction). La distinction entre l'origine de ces effets n'est pas rigoureuse, les diagrammes représentant les différentes interactions possibles pour passer d'un atome dans un état initial donné à un état final donné devant être traités de façon globale, mais elle est pratique pour les expérimentateurs afin de décrire les phénomènes observés. 
Dans le cas de la photoionisation directe de l'état fondamental des atomes, l'intensité de la raie principale reste toujours supérieure à celle des satellites de corrélation, encore que l'intensité relative de ceux-ci puisse atteindre des valeurs assez élevées telles que, par exemple $70 \%$ dans le cas du baryum photoionisé en couche 4d[74]. Mais la production d'états satellites est, en général, un processus d'intensité plus faible, demandant des flux de photons encore plus importants, et ce d'autant plus que l'on voudrait en déterminer la dépendance angulaire. Pour un atome dans un état excité, un autre type de satellites peut apparaître; les satellites dit "shake down", à une énergie cinétique supérieure à celle de la raie principale: dans le processus qui leur donne naissance, l'énergie đânsmise à l'atome par l'excitation laser est restituée au photoélectron qui augmente dor son énergie cinétique de la même quantité. Cet effet a effectivement observé dans le sodium[37, 62] (voir ci-dessous). Rappelons enfin que le deuxième électron peut aussi être éjecté dans le continuum, reflétant l'existence du processus de photoionisation double. Mais ce dernier phénomène n'a pas encore été mis en évidence, ni donc a fortiori étudié, dans un atome excité.

\subsection{Premières études}

Nous avons déja souligné la plus grande difficulté d'étudier les processus de photoionisation directe, étant donné leur probabilité plus faible qui exige donc des flux de photons nettement plus importants que pour les processus résonants. Une section efficace de photoionisation d'un atome en couche interne dépasse rarement 10 à $20 \mathrm{Mb}$, alors que l'on connaît des excitations résonantes en couche interne dont la section efficace peut atteindre $3000 \mathrm{Mb}$. [75]. Quant à la photoionisation directe d'électrons externes, si elle peut être intense dans les quelques électron-volts au dessus de la limite d'ionisation - région d'énergie qui est, incidemment, tellement plus facilement

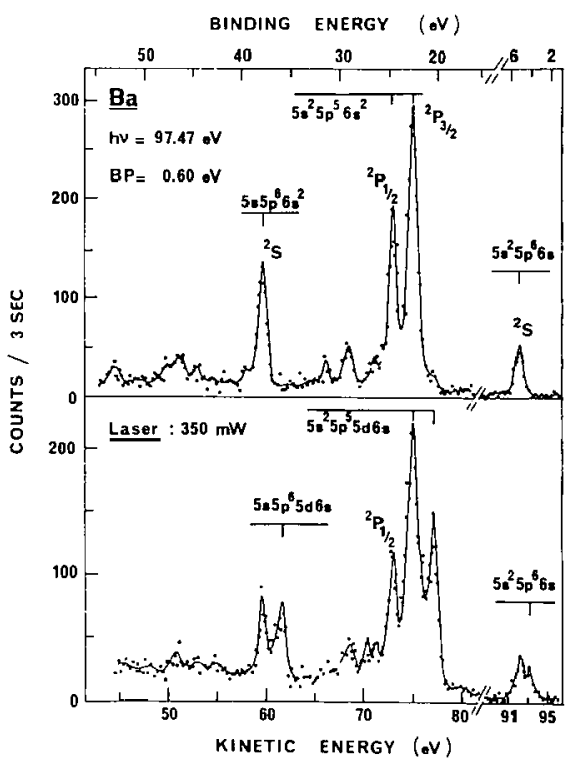

Fig. 9 - Spectres de photoélectrons dus d la photoionisation en couches $5 s, 5 p, 5 d$, et $6 s$ d'atomes de baryum d l'état fondamental (partie haute) etdans les états excités $5 s^{2} 5 p^{6} 6 s 5 d^{1,3} \mathrm{D}$ (partie basse) avec des photons de $97,5 \mathrm{eV}$. explorée avec les lasers qu'il ne semble pas judicieux, ni nécessaire d'y employer le rayonnement synchrotron[76]- leurs sections efficaces atteignent difficilement $0,1 \mathrm{Mb}$ (sauf conditions spéciales[36,69] dont nous évoquerons un exemple ci-dessous) dans le domaine des "hautes" énergies (15-150 eV) actuellement concerné par ces expériences couplant l'utilisation des rayonnements synchrotron et laser(s).

Avant de pouvoir disposer, vers la fin des années 80 , des nouvelles sources plus intenses de rayonnement synchrotron (Super ACO, onduleur sur BESSY), si la photoionisation directe dans le continuum a été observée dès l'origine[1, 42,43], un seul cas avait pu être étudié, grâce à la particularité déja mentionnée dans un précédent paragraphe: la désexcitation rapide de l'état optiquement excité ${ }^{1} \mathrm{P}_{1}$ du baryum vers les états métastables $1,3 \mathrm{D}_{2}$, permettant d'atteindre parfois des densités relatives d'atomes dans ces états excités voisines de $100 \%$ (cf figure 5). Ceci a permis d'analyser quantitativernent [36, 39], les spectres de photoélectrons dus à la photoionisation, en couches internes et en couche externe, de l'atome de baryum excité dans les états $5 s^{2} 5 p^{6} 6 s 5 d 1,3 \mathrm{D}$. Nous en montrons un exemple[2,56] dans la Figure 9, obtenue à une énergie de photons de $97,5 \mathrm{eV}$, avec une 
densité relative d'atomes excités qui était ici d'environ $50 \%$. Chaque raie principale est dédoublée ; l'effet est particulièrement clair pour la raie $5 \mathrm{~s}$, plus difficile à analyser pour les raies $5 p$ déjà dédoublées dans l'état fondamental par interaction spin-orbite. Il est intéressant de noter que l'énergie de liaison des électrons internes est plus faible dans le baryum excité que dans l'état fondamental, ce qui traduit un effet d'écran externe augmenté à la suite de l'excitation laser. Cette variation est de sens opposé à ce qui a été observé dans le sodium (sous-couches 2p et 2s) [34, 61, 87], et, plus généralement, dans les alcalins[62]. Des satellites de corrélation sont également observés. Notons au passage que la production des états satellites peut également s'observer dans l'autoionisation et que nous en avons vu un exemple particulièrement significatif à la suite de l'excitation en couche $5 \mathrm{p}$ du baryum excité (revoir figure 5).

La variation, en fonction de l'énergie des photons, des sections efficaces de photoionisation en sous-couches $5 d, 5 s, 5 p$ et $6 \mathrm{~s}$ a pu être mesurée[36, 67, 69]. La Figure 10 montre les résultats obtenus pour les sous-couches $5 \mathrm{~s}$ et $5 \mathrm{p}$. Comme pour toute mesure de sections efficaces dans un atome excité, la densité relative des atomes excités a été déterminée par la réduction d'intensité d'une raie de photoélectrons due à la photoionisation de l'état fondamental lorsque le laser irradie la vapeur, la raie 5s dans la figure 9 à une énergie cinétique de $59 \mathrm{eV}$. $\mathrm{La}$ valeur absolue des sections efficaces a été déterminée, en l'absence de mesures absolues de la section efficace totale de photoabsorption, par normalisation aux valeurs théoriques de la section efficace 5p calculées dans le modèle LDRPA[36]. On remarque, pour les deux souscouches - comme pour la sous-couche $5 \mathbf{d}$ - une augmentation importante de la section efficace qui est corrélée à une augmentation parallèle de la section efficace de photoionisation en couche $4 \mathrm{~d}$ à partir des seuils d'ionisation $4 \mathrm{~d}(98,4 \mathrm{et} 101.0 \mathrm{eV})$. L'existence de fortes corrélations inter-voies ( $4 d, 5 s, 5 p)$ avait déjà été mesurée pour des atomes dans l'état fondamental[ $34,77,78]$, mais c'était la première fois qu'elles étaient observées dans un atome excité, souligant l'importance des effets de corrélation pour le comportement de propriétés apparemment monoélectroniques: dans cette région d'énergie de photons voisine de $100 \mathrm{eV}$, les résultats du modèle à un électron[79] donnent, en effet, une décroissance continue et lente de ces sections efficaces (courbes en pointillés dans la figure 10).
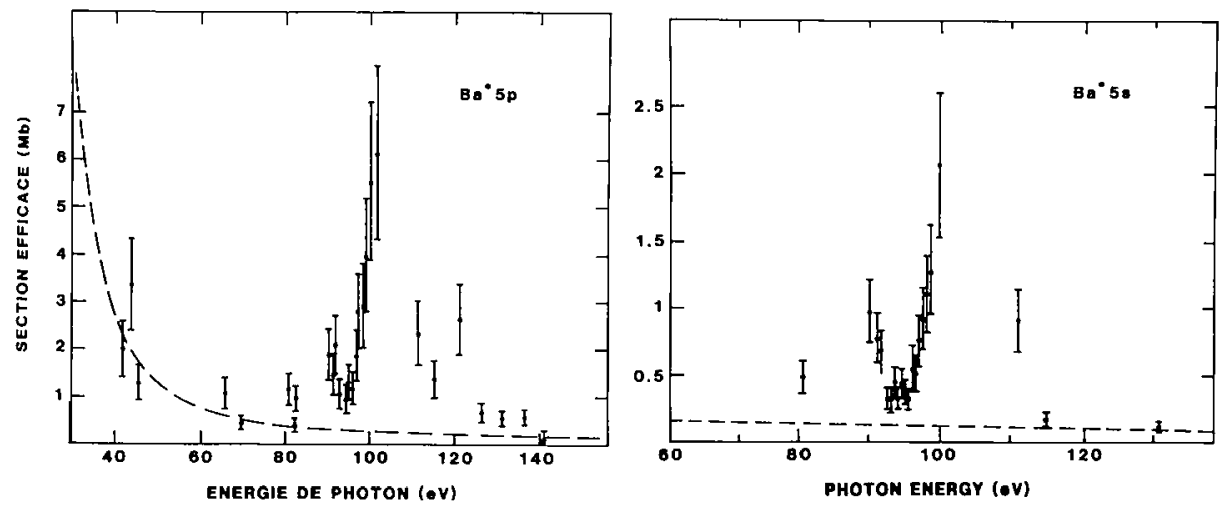

Fig. 10 - Variation, en fonction de l'énergie des photons, des sections efficaces de photoionisation en sous-couche $5 p$ (d gauche) et $5 s$ (à droite) dans l'atome de baryum excité dans les états $6 s 5 d^{1.3}$ D (Bizau et al., 1987, réf. 69).

\section{2 Mise en évidence d'un nouveau phénomène : l'augmentation importante des effets de corrélation dans les atomes excités.}

Malgré les densités beaucoup plus faibles d'atomes excités, il avait été possible, dès l'origine[2, 42,43], d'observer dans le sodium une raie de photoélectrons due à la photo- 
ionisation directe en couche $2 p$ du sodium excité dans l'état $2 p^{6}{ }^{6}{ }^{2} P_{3 / 2}$. Ce premier spectre, obtenu en 1981, est rappelé dans la Figure 11a (partie gauche). Malgré l'optimisation rapide des conditions expérimentales (cf. Figure 3), il fallut attendre Super ACO pour pouvoir mesurer de façon précise $(5 \%)$ les premières sections efficaces de photoionisation en couche interne, pour les alcalins (sodium, potassium) [37, 61-63], sections efficaces que des calculs, effectués dans la théorie des perturbations à $\mathrm{N}$-corps (MBPT), prévoyaient être les mêmes que dans l'état fondamental[80, 81]. Dans la Figure $11 \mathrm{~b}$ (partie droite), nous montrons un exemple de spectre de photoélectrons dus à la photoionisation du sodium dans l'état fondamental (partie haute) et dans l'état excité (partie basse) obtenus[37, 62] avec Super-ACO. Par rapport aux meilleurs résultats obtenus avec ACO, l'amélioration est spectaculaire, avec un bruit de fond presque nul et un rapport signal/bruit inégalé. Dans l'état fondamental, même des satellites de corrélation du deuxième ordre $(\Delta \mathrm{n}=2)$ sont mis en évidence. Pour l'état excité, il a été possible, par une procédure de soustraction-normalisation, de déterminer le spectre pur de photoélectron montré ici, bien que la densité d'atomes dans l'état excité n'ait pas dépassé $15 \%$. La différence qualitative entre les deux états atomiques est évidente à l'observation des spectres. Elle démontre une augmentation spectaculaire de l'intensité relative des effets de corrélation de l'état excité, augmentation qui se fait au détriment du processus simple de photoionisation. Ces résultats n'avaient pas été prévus par la théorie.
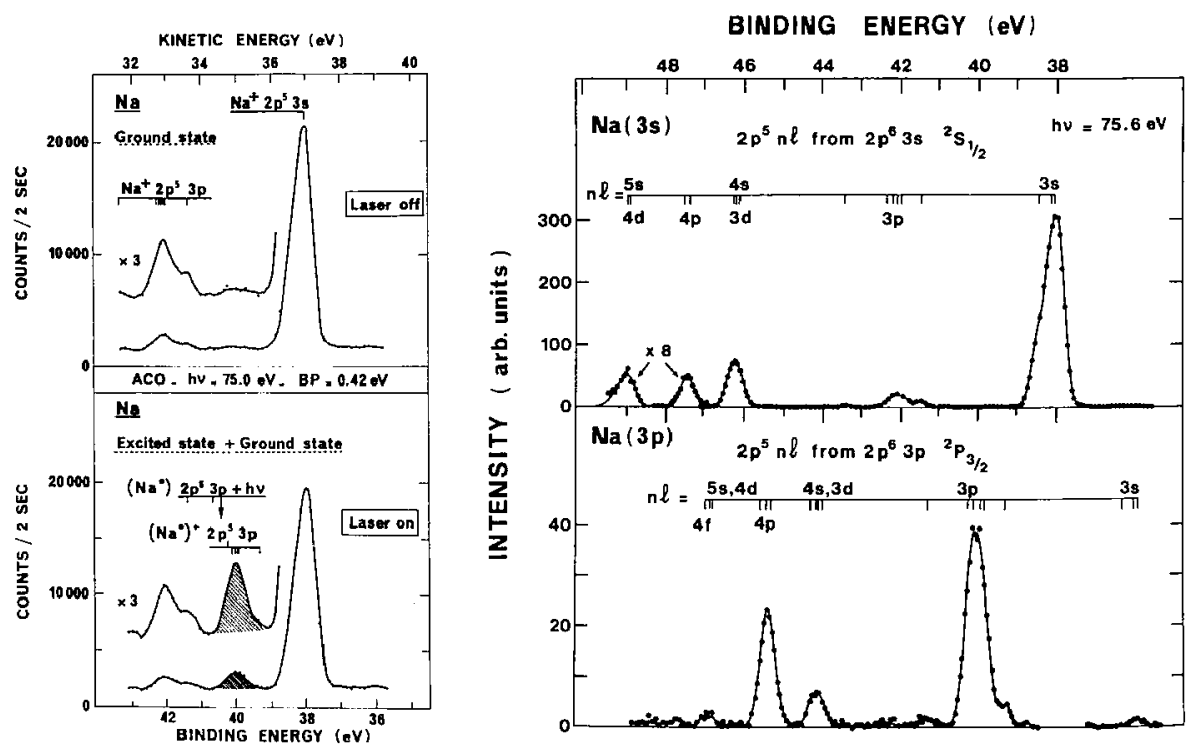

Fig. 11 - a) A gauche: spectres de photoélectrons observés durant la première expérience avec $A C O$, en 1981. Ils sont dus a la photoionisation directe, en sous-couche $2 p$, d'atomes de sodium dans l'état fondamental (partie haute) et d'un mélange d'atomes de sodium dans l'état fondamental (90\%) et dans l'état excité $2 p^{6} 3 p^{2} P_{3 / 2}(10 \%)$. La raie hachurée, à une énergie de liaison de $40 \mathrm{eV}$, était la signature de la photoionisation des atomes excités (Bizau et al., 1981, réf. 1). b) A droite: spectres de photoélectrons du sodium à l'état fondamental (partie haute) et dans l'état excité (partie basse), engistrés avec le rayonnement de Super ACO (Cubaynes et al., 1989, réf. 37 et 1991, réf. 62).

L'analyse quantitative des spectres tels que ceux présentés dans la figure $11 \mathrm{~b}$ a montré que cette section efficace de photoionisation simple en sous-couche interne $2 \mathrm{p}$ est réduite de $20 \%$ environ dans l'état excité. Ce résultat est montré Figure 12. Par contre, l'intensité relative des satellites de corrélation produits par effet shake up (configurations électroniques $2 p^{5} 4 p$ 
dans l'état excité, $2 \mathrm{p}^{54 \mathrm{~s}}$ ou $2 \mathrm{p}^{5} 3 \mathrm{~d}$ dans l'état fondamental) est doublée dans l'état excité (40\%) par rapport à l'état fondamental $(20 \%)$, comme l'illustre la figure 13. Par comparaison, l'intensité relative des satellites de corrélation produits par effet shake up conjugué (configurations électroniques $2 p^{5} 3 \mathrm{~d}$ ou $2 \mathrm{p}^{5} 4 \mathrm{~s}$ dans l'état excité, $2 \mathrm{p}^{5} 3 \mathrm{p}$ dans l'état fondamental) est à peu près la même dans les deux états, décroissant lentement d'environ $12 \%$ à $60 \mathrm{eV}$ jusqu'à 8 $\%$ vers $110 \mathrm{eV}$. Un comportement semblable a été ensuite observé pour la photoionisation en couche $2 \mathrm{~s}$ de l'atome de sodium excité dans l'état $3 \mathrm{P}_{3 / 2}$ et en couche $3 \mathrm{p}$ de l'atome de potassium excité dans l'état $4 \mathrm{P}_{3 / 2}$. Ces résultats nouveaux et imprévus furent expliqués en considérant le phénomène de contraction des orbitales dans l'état final, par suite de la variation d'une unité de la charge du coeur électronique, qui impliquait un recouvrement différent des fonctions d'onde initiales et finales dans l'état fondamental et dans l'état excité $[37,61,87]$. Globalement, la section efficace totale de photoabsorption en couche interne ne varie pas, le phénomène se traduisant seulement par un transfert de force d'oscillateur des transitions à un électron vers les transitions à deux électrons. Il fut vérifié ultérieurement, mais encore seulement dans le cas du lithium excité (état $1 s^{2} 2 \mathrm{p}^{2} \mathrm{P}_{3 / 2}$ ), que la théorie pouvait reproduire le comportement découvert expérimentalement[88]. Des mesures préliminaires sur le lithium excité[83] semblent indiquer le même comportement que celui observé pour le sodium et le potassium. Rappelons que, dans le cas des processus résonants, nous avons trouvé une force d'oscillateur pour la somme des transitions simples $2 \mathrm{p} \rightarrow 3 \mathrm{~s}$ d'environ 0,12 dans l'état fondamental $2 p^{6} 3 s^{2} S_{1 / 2}$ et 0,18 dans l'état excité $2 p^{6} 3 p^{2} \mathrm{P}_{3 / 2}$ du sodium[34, 44].

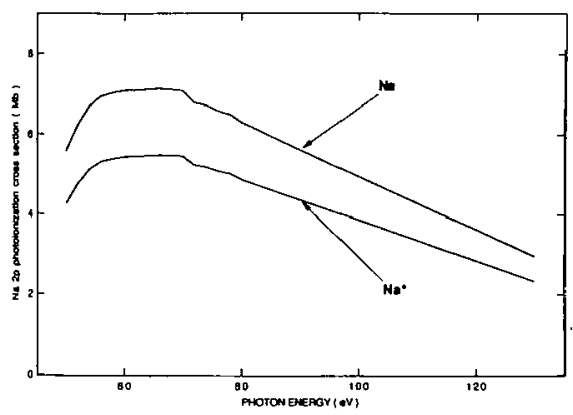

Fig. 12 - Variation de la section efficace de photoionisation simple en sous couche $2 p$ du sodium d l'état fondamental (courbe du haut) et dans l'état excité $2 p^{6} 3 p{ }^{2} P_{3 / 2}$ (courbe du bas) entre 50 et $130 \mathrm{eV}$ (Bizau et al., 1992, réf. 82).

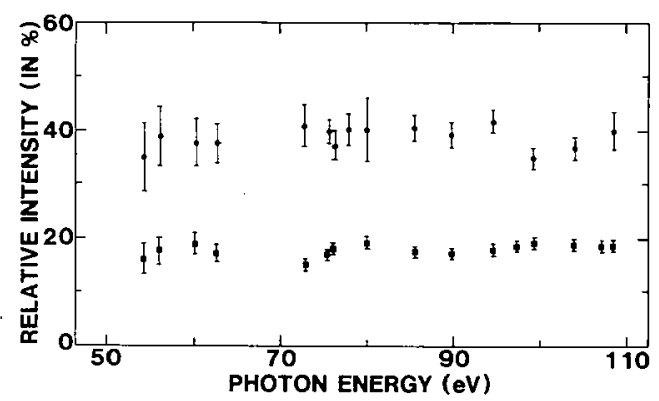

Fig. 13 - Intensité relative des satellites shake up produits dans la photoionisation du sodium à l'état fondamental (carrés noirs, en bas) et dans l'état excité $3 p$ (ronds noirs, en haut) (Cubaynes et al., 1989, réf. 39).

Enfin, parmi les tout derniers résultats obtenus, nous montrerons un exemple de spectre de photoélectrons résolu angulairement mesuré[84] avec le dispositif indiqué dans la figure 4. La photoionisation en sous-couche $2 p$ du sodium a été mesurée pour 6 valeurs différentes de l'angle $\varphi$, dans l'état fondamental et dans l'état excité. L'analyse des spectres, dont un exemple est montré Figure 14, est en cours. Elle implique des mesures complémentaires, longues et minutieuses, avec différentes raies de photoélectrons et d'électrons Auger dans les gaz rares $(\mathrm{He}, \mathrm{Ar}, \mathrm{Xe})$ afin de mesurer avec précision la polarisation du rayonnement synchrotron, y compris la rotation de l'ellipse de polarisation induite par réflection sur les différentes surfaces optiques, la transmission et l'efficacité du CMA en fonction de l'angle $\varphi$. Notons que, si un onduleur pouvait être utilisé pour ces mesures (et il devra l'être pour l'étude angulaire des satellites de corrélation), cette procédure d'étalonnage sera très simplifiée, le rayonnement synchrotron émis par un onduleur[85] étant pratiquement polarisé linéairement à $100 \%$ dans le 


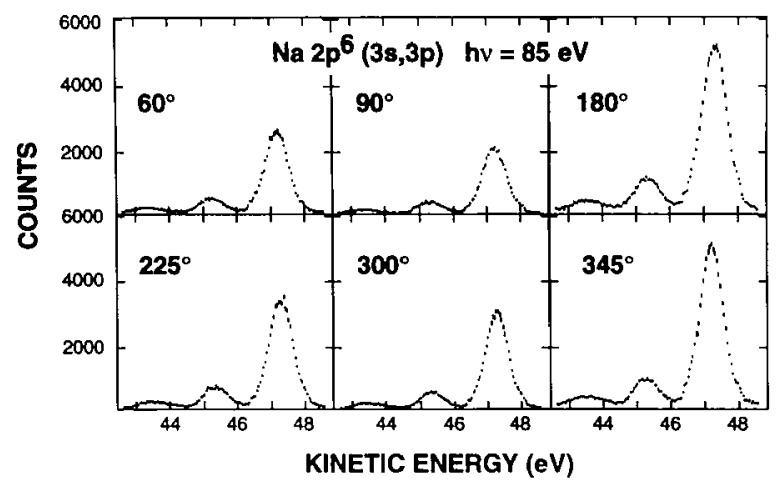

Fig. 14 - Spectres de photoélectrons résolus angulairement, mesurés pour six valeurs différentes de l'angle $\varphi$, provenant de la photoionisation en sous-couche $2 p$ du sodium dans son état fondamental (raie principale, à $47 \mathrm{eV}$ d'énergie cinétique, raies satellites, en dessous de $44 \mathrm{eV}$ ) et dans l'état excité $3 p$ (raie principale, d $45 \mathrm{eV}$ ) (Rouvellou et al., 1992, réf. 84).

plan de l'orbite (98\% pour les expériences de distribution angulaire des électrons émis dans l'autoionisation des états 1 snln'l' du lithium[39]).

\section{CONCLUSION}

Dans une précédente revue écrite avant la mise en fonctionnement des nouvelles sources de rayonnement synchrotron[86], nous avions dressé la liste des processus qu'une amélioration sensible des performances du rayonnement synchrotron devait permettre d'étudier. Ceux-ci comprenaient, entre autres:

- L'étude de processus à faibles sections efficaces

- La possibilité de travailler avec des densités d'atomes plus faibles

- L'amélioration de la résolution

- Le raccourcissement du temps d'enregistrement des spectres afin de s'affranchir au maximum des instabilités du laser.

- L'analyse angulaire des électrons émis

- La synchronisation d'un laser pulsé avec l'émission pulsée du rayonnement synchrotron et avec le même taux de répétition, pour la physique moléculaire.

A l'excepuion de la dernière de ces propositions, dont la réalisation est activement en cours d'étude, toutes les autres ont été effectivement accomplies en l'espace de cinq années, soulignant les progrès rapides des études en ce domaine lorsque de meilleures sources de rayonnement sont mises à disposition des chercheurs. En France, la prochaine étape sera la construction du nouvel anneau de stockage SOLEIL en cours d'étude, qui devrait permettre d'utiliser régulièrement un onduleur dans des expériences qui, comme toutes celles décrites cidessus, sont très demandeuses de performances accrues pour les sources de rayonnement synchrotron et sont rapidement en état d'exploiter efficacement les nouveaux outils mis à leur disposition.

\section{REMERCIEMENTS}

Les auteurs souhaitent remercier d'abord les collaborateurs qui leur ont permis de commencer ce programme d'expérience, en particulier J.-L. Picqué et J.-L. LeGouët, puis ceux avec lesquels ils ont continué tout au long des années, notamment D. L. Ederer, B. Carré, T.J. 
Morgan, F. Gounand, M. Richter, enfin les nouveaux, jeunes et enthousiastes, B. Rouvellou et L. Journel. Ils expriment toute leur reconnaissance à $M$. Berland qui a assuré la mise en page de ce texte.

\section{REFERENCES}

[1] BIZAU, J -M, WULLEUMIER, F, J, LE GOUET, J -L, EDERER, D, L, PICQUE, J -L, et DHEZ, P, XI ICPEAC, Abstracts of post-post deadline papers, ed. S. Datz, 1981, p. 1.

[2] WUILEUMIER, F J, dans: "X-ray and Atomic Inner-Shell Physics", ed. B. Crasemann, AIP Conf. Proc. No94 (American Institute of Physics: New York) 1982, p. 615.

[3] MEHLMAN-BALLOFET, G, et ESTEVA, J -M, Astrophys. 157 (1969) 945.

[4] ESTEVA, J -M, MEHLMAN-BALLOFET, G, et ROMAND, J, J. Quant. Spectr. Radiat. Transf. 12.(1972) 1291.

[5] MEHLMAN, G, et ESTEVA, J -M, C. R. Acad. Sci. 276 (1973) 1973.

[6] ESTEVA, J -M, et MEHLMAN, G, Astrophys. J. 193 (1974) 747.

[7] CARILLON, A, JAEGLE, P, et DHEZ, P, Phys. Rev. Lett. 25 (1970) 140.

[8] JAEGLE, P, CARILLON, A, JAMELOT, G, SUREAU, A, et DHEZ, P, Phys. Rev. Lett. 33 (1974) 1070.

[9] CARILLON, A, et al. Phys. Rev. Lett. 68 (1992) 2917.

[10] CARROLL, P, K, et KENNEDY, E, T, Phys. Rev. Lett. 38 (1977) 1068.

[11] CARROLL, P, K, KENNEDY, E, T, et O'SULLIVAN, G, Appl. Opt. 19 (1980) 1434.

[12] JANITTI, E, NICOLOSI, P, et TONDELLO, G, Physica Scripta 36 (1987) 93.

[13] JANITTI, E, NICOLOSI, P, et TONDELLO, G, dans: "X-ray and Inner Shell Processes", eds. T. A. Carlson, M. O. Krause et S. T. Manson, AIP Conf. Proc. N²15 (Amer. Inst. Phys. : New York) 1990 , p. 672.

[14] COSTELLO, J, T, KENNEDY, E, T, SONNTAG, B, et ClARCK, C, W, Phys. Rev. A 43 (1991) 1441.

[15] COSTELLO, J, T, MOSNIER, J, P, KENNEDY, E, T, CARROLL, P, K, et O'SULLIVAN, G, Physica Scripta T 34 (1991) 77.

[16] LUCATORTO, T, B, et McILRATH, T, J, Phys. Rev. Lett. 37 (1976) 428.

[17] McILRATH, T, J, et LUCATORTO, T, B, Phys. Rev. Lett. 38 (1977) 1390.

[18] LUCATORTO, T, B, McIlRATH, T, J, SUGAR, J, et YOUNGER, S, M, Phys. Rev. Lett.47 (1981) 1124.

[19] SUGAR, J, LUCATORTO, T, B, McIlRATH, T, J, et WEISS, A, W, Opt. Lett. 4 (1979) 109.

[20] CROMER, C, L, BRIDGEIS, J, M, ROBERTS J, M, et LUCATORTO, T, B, Appl. Opt. 24 (1985) 2996.

[21] SONNTAG, B, F, CROMER, C, L, BRIDGEIS, J, M, McILRATH, T, J, et LUCATORTO, T, B, dans: "Short Wavelength Coherent Radiation: Generation and Applications", AIP Conf. Proc. N 147 (Amer. Inst. Phys.: New York) 1986, p. 412.

[22] COOPER, J, W, CLARCK, C, W, CROMER, C, L, LUCATORTO, T, B, SONNTAG, B, F, et COSTELLO, J, T, Phys. Rev. A 39 (1989) 6074.

[23] McIlRATH, T, J, Appl. Phys. Lett. 15 (1969) 41.

[24] BRADLEY, D, J, EWARD, P, NICHOLAS, J, W, et SHAW, J, R, D, J. Phys. B 6 (1973) 1594.

[25] KLUCHAREV, A, N, et RYAZANOV, N, S, Opt. Spectr. 32 (1972) 686.

[26] KLUCHAREV, A, N, et SEPMAN, V, Yu, Opt. Spect. 38 (1975) 712.

[27] STEBBINGS, R, F, DUNNING, F, B, TITTEL, F, K, et RUNDEL, R, D, Phys. Rev. Lett. 30 (1973) 815.

[28] RUNDEL, R, D, DUNNING, F, B, GOLDWIRE, Jr, M, C, et STEBBINGS, R, F, J. Opt. Soc. Amer. 65 (1975) 628

[29] HANSEN, J, C, DUNCANSON, J, A, Jr, CHIEN, R-L, et BERRY, R, S, Phys. Rev. A 21 (1980) 222.

[30] CHIEN, R-L, MULLINS, O, C, et BERRY, R, S, Phys. Rev. A 28 (1983) 2078.

[31] SIEGEL, A, GANZ, J, BUSSERT, W, et HOTOP, H, J. Phys. B 16 (1983) 2945.

[32] MULLINS, O, C, CHIEN, R-L, HUNTER, J, E, III, KELLER, J, S, et BERRY, R, S, Phys. Rev. A 31 (1985) 321.

[33] KERLING, C, BOWERING, N, et HEINZMANN, U, J. Phys. B 23 (1990) L629.

[34] BIZAU, J -M, WUILLEUMIER, F, J, EDERER, D, L, KELLER, J -C, LEGOUET, J -L, PICQUE, J, L, CARRE, B, et KOCH, P, M, Phys. Rev. Lett. 55 (1985) 1281. 
[35] FERRAY, M, GOUNAND, F, D'OLIVEIRA, P, FOURNIER, P, R, CUBAYNES, D, BIZAU, J -M, MORGAN, T, J, et WUILLEUMIER, F, J, Phys. Rev. Lett. 59 (1987) 2040.

[36] BIZAU, J -M, CUBAYNES, D, GERARD, P, WUILLEUMIER, F, J, PICQUE, J, L, EDERER, D, L, et WENDIN, G, Phys. Rev. Lett. 57 (1986) 306.

[37] CUBA YNES, D, BIZAU, J -M, WUILLEUMIER, F, J, CARRE, B, et GOUNAND, F, Phys. Rev. Lett. 63 (1989) 2460.

[38] MEYER, M, MULLER, B, NUNNEMAN, A, PRESCHER, T, VON RAVEN, E, RICHTER, M, SCHMIDT, M, SONNTAG, B, F, et ZIMMERMANN, P, Phys. Rev. Lett. 59 (1987) 2963.

[39] PAHLER, M, LORENZ, C, VON RAVEN, E, RUDER, J, SONNTAG, B, F, MULLER, B, R, SCHULZE, M, STAIGER, H, KABACHNIK, N, M, et ZIMMERMANN, P, Phys. Rev. Lett. 68 (1992) 2285.

[40] TREMBLAY, J, LARZILLIERE, M, COMBET FARNOUX, F, et MORIN, P, Phys. Rev. A 38 (1988) 3804.

[41] LEE, J, dans: "Combustion Dynamics Facility", Lawrence Berkeley Laboratory and Sandia National Laboratories (Berkeley) 1990, PUB-5284.

[42] BIZAU, J-M, WUILLEUMIER, F, J, DHEZ, P, EDERER, D, L, PICQUE, J-L, LEGOUET, J -L, et KOCH, P, M, dans: "Laser Techniques for the Extreme Ultraviolet Spectroscopy", eds. T. J. Mcilrath et R. Freeman, AIP Conf. Proc. Nº0 (Amer. Inst. Phys. : New York) 1982, p. 331.

[43] WUILLEUMIER, F, J, J. Physique, 43 (1982) C2-347.

[44] WUILLEUMIER, F, J, dans: "Laser Techniques in the Extreme Ultraviolet", eds. S. E. Harris et T. B. Lucatorto, AIP Conf. Proc. No119 (Amer. Inst. Phys.: New York) 1984, p. 220.

[45] WUILlEUMIER, F, J, EDERER, D, L, et PICQUE, J -L, Adv. At. Molec. Phys. 23 (1987) p. 197.

[46] EDERER, D, L, RUBENSON, J, E, MUELLER, D, R, SHUKER, R, O'BRIEN, W, L, JAI, J, DONG, R, Y, CALCOTT, D, A, CARR, G, L, WILLIAMS, G, P, HIRSCHMUGL, C, J, ETEMAD, S, INAM, A, et TANNER, D, B, Nucl. Instr. Meth. A 379 (1992) 250.

[47] LARSEN, P, K, BERS, W, A, M, BIZAU, J-M, WUILLEUMIER, F, J, KRUMMACHER, S, SCHMIDT, V, et EDERER, D, L, Nucl. Instr. Meth. 195 (1982) 245.

[48] WUILLEUMIER, F, J, Atom. Phys. 7 (1981) 493.

[49] CARRE, B, D'OLIVEIRA, P, FERRAY, M, FOURNIER, P, R, GOUNAND, F, CUBA YNES, D, BIZAU J -M, et WULLLEUMIER, F, J, Z. f. Phys. 15 (1990) 117.

[50] SCHMWDT, V, Phys. Lett. 45A (1973), 63.

[51] CUBAYNES, D, BIZAU, J -M, MORGAN, T, J, WULLEUMIER, F, J, FERRAY, M, GOUNAND, F, D'OLIVEIRA, P, et FOURNIER, P, R, J. Physique 48 (1987) C9-513.

[52] FISCHER, A, et HERTEL, I, V, Z. Phys. A 304 (1982) 103.

[53] MEYER, M, PAHLER, M, PRESCHER, Th, VON RAVEN, E, RICHTER, M, SONNTAG, B, F, BAIER, S, FIEDLER, W, MULLER, B, R, SCHULZE, M, et ZIMMERMANN, P, Physica Scripta T 31 (1990) 28.

[54] PAHLER, M, Dissertation, Université d'Hambourg, 1991.

[55] BIZAU, J -M, Thèse de Doctorat d'Etat, Orsay, 1987.

[56] BIZAU, J -M, CARRE, B, DHEZ, P, EDERER, D, L, GERARD, P, KELLER, J -C, KOCH, P, LEGOUET, J -L, PICQUE, J -L, WENDIN, G, et WULLLEUMIER, F, J, XII ICPEAC, Abstracts of Contributed Papers, eds. J. Eichler, W. Fritsch, I. V. Hertel, N. Stolterfoht et N. Wille (Berlin) 1983, p. 27.

[57] LEGOUET, J -L, PICQUE, J -L, WUILLEUMIER, F, J, BIZAU, J -M, DHEZ, P, KOCH, P, M, et EDERER, D, L, Phys. Rev. Lett. 48 (1982) 600.

[58] NUNNEMANN, A, PRESCHER, Th, RICHTER, M, SCHMIDT, M, SONNTAG, B, F, WETZEL, H, E, et ZIMMERMANN, P, J. Phys. B 18 (1985) L337.

[59] BIZAU, J -M, CUBAYNES, D, ROUVELLOU, B, et WULLLEUMIER, F, J, IVth ECAMP, Europhys. Conf. Abstr. 16 B (1992) part I, p. 173.

[60] CUBAYNES, D, BIZAU, J -M, MORGAN, T, J, NOVAK, J, PAHLER, M, ROUVELLOU, $B$, SCHLACHTER, F et WUILLEUMIER, F, J, IVth ECAMP, Europhys. Conf. Abstr. 16 B (1992) part I, p. 174.

[61] RICHTER, M, BIZAU, J -M, CUBAYNES, D, MENZEL, T, WUILLEUMIER, F, J, et CARRE, B, Europhys. Lett. 12 (1990) 35. 
[62] WUILLEUMIER, F, J, CUBAYNES, D, et BIZAU, J -M, dans: "Atomic and Molecular Physics", eds. C. Cisneros, I. Alvarez et T. J. Morgan (World Scientific: Singapore) 1991, p. 474.

[63] CUBAYNES, D, BIZAU, J-M, MORGAN, T, J, CARRE, B, et WULLEUMIER, F, J, 10th VUV, Abstracts, Vacuum Ultraviolet Radiation Physics, eds. F. J. Wuilleumier, I. Nenner et Y. Petroff (1992) p.TU79.

[64] CUBAYNES, D, BIZAU, J -M, MARINELLI, C, MORGAN, T, J, NOVAK, J, PAHLER, M, ROUVELLOU, B, SCHLACHTER, F, BERRAH-MANSOUR, $N$, et WUILLEUMIER, F, J, 10th VUV, Abstracts, Vacuum Ultraviolet Radiation Physics, eds. F. J. Wuilleumier, I. Nenner et Y. Petroff (1992) p.TU80.

[65] PRESCHER, Th, Thèse de l'Université de Hamburg, 1988.

[66] CUBA YNES, D, BIZAU, J, M, GERARD, P, WUILLEUMIER, F, J, PICQUE, J -L, CARRE, B, ROUSSEL, F, et SPIESS, G, VUV 8, Vacuum Ultraviolet Radiation Physics, Book of Abstracts, vol. III, Post deadline papers, ed. P,O, Nilssffahalmers University of Technology: Göteborg) 1986, p. 658.

[67] CUBA YNES, D, Thèse, Université Paris-Sud, Orsay, 1987.

[68] MEYER, M, MULLER, B, NUNNEMANN, A, PRESCHER, Th, VON RAVEN, E, RICHTER, M, SCHMDT, M, SONNTAG, B, F, et ZIMMERMANN, P, J. Physique 48 (1987) C9-551.

[69] BIZAU, J -M, CUBAYNES, D, GERARD, P, WUILLEUMIER, F, J, et PICQUE, J -L, J. Physique, 48 (1987) C9-491.

[70] MEYER, M, PRESCHER, Th, VON RAVEN, E, RICHTER, M, SONNTAG, B, F, MULLER, B, R, FIEDLER, W, et ZIMMERMANN, J. Physique 48 (1987) C9-547.

[71] AMUSIA, M; Ya, dans: "Vacuum Ultraviolet Radiation Physics", eds. F. J. Wuilleumier, I. Nenner et J. Petroff (World Scientific: Singapore) 1992, p.1

[72] MARTIN, R, L, et SHIRLEY, D, A, Phys. Rev. A 13 (1976) 1475.

[73] WUILLEUMIER, F, J, éditeur, "Photoionization and Other Probes of Electron Correlations", (Plenum Press: New York) 1976.

[74] BIZAU, J -M, CUBA YNES, D, GERARD, P, et WULlEUMIER, F, J, Phys. Rev. A 40 (1989) 3002.

[75] LYON, I, C, PEART, B, WEST, J, B, et DOLDER, K, J. Phys. B 19 (1986) 4137.

[76] PRESSES, J, M, BURKHARDT, C, E, COREY, R, L, EARSOM, D, L, DAULTON, T, L, GARVER, W, P, LEVENTHAL, J, J, MSEZANE, A, Z, et MANSON, S, T, Phys. Rev. A 32 (1985) 1264.

[77] WEST, J, B, WOODRUFF, P, R, CODLING, K, et HOULGATE, R, G, J. Phys. B 9 (1976) 407

[78] ADAM, M, Y, WUILLEUMIER, F, J, SANDNER, N, KRUMMACHER, S, SCHMIDT, V, et MEHLHORN, W, Jap. J. Appl. Phys. 17-2 (1978) 43.

[79] THEODOSIOU, C, E, Phys. Rev. A 33 (1986) 2164.

[80] CHANG, T, N, et KIM, Y, S, J. Phys. B 15 (1982) L835.

[81] CHANG, T, N, et KIM, Y, S, AIP Conf. Proc. Nº94, ed. B. Crasemann (Amer. Inst. Phys.: New York) p.633.

[82] BIZAU, J -M, CUBAYNES, D, et WUILLEUMIER, F, J, dans; "Vacuum Ultraviolet Radiation Physics", eds. F. J. Wuilleumier, I. Nenner et Y. Petroff (World Scientific: Singapore) 1992, p.

[83] JOURNEL, L, et al., à paraître.

[84] ROUVELLOU, B, et al. à paraître

[85] KRAUSE, M, O, WHITFIELD, S, B, CALDWELL, C, D, WU, J, Z, VAN DER MEULEN, P, DE LANGE, C, A, et HANSEN, R, W, C, J. Electr. Spectr Rel. Phen. 58 (1992) 79.

[86] CUBAYNES, D, BIZAU, J -M, WUILLEUMIER, F, J, EDERER, D, L, PICQUE, J, L, CARRE, B, FERRAY, M, GOUNAND, F, J. Physique 48 (1987) C9-453.

[87] CUBAYNES, D, BIZAU, J-M, CARRE, B, et WUILLEUMIER, F, J, dans: "The Physics of Electronic and Atomic Collisions", eds. A. Dalgarno, R. S. Freund, P. M. Koch, M. S. Lubbel et T. B. Lucatorto, AIP Conf. Proc. N²05 (Amer. Inst. Phys.:New York) 1989, p. 224.

[88] FELFLI, Z, et MANSON, S, T, Phys. Rev. Lett. 68 (1992) 1687.

[89] BAIER, S, FIEDLER, W, MULLER, B, SCHULZE, M, ZIMMERMANN, P, MEYER, M, PAHLER, M, PRESCHER, Th, VON RAVEN, E, RICHTER, M, RUDER, J, et SONNTAG, B, F, J. Phys. B 25 (1992) 923. 